\title{
Friction factor correlations for laminar, transition and turbulent flow in smooth pipes
}

\author{
By D.D. JOSEPH ${ }^{\dagger}$ AND B.H. YANG ${ }^{\dagger}$ \\ ${ }^{\dagger}$ Department of Aerospace Engineering and Mechanics, University of Minnesota, Minneapolis, 55455 \\ *Department of Mechanical and Aerospace Engineering, University of California, Irvine, 92617 \\ July, 2008
}

In this paper we derive an accurate composite friction factor vs. Reynolds number correlation formula for laminar, transition and turbulent flow in smooth pipes. The correlation is given as a rational fraction of rational fractions of power laws which is systematically generated by smoothly connecting linear splines in $\log$ - log coordinates with a logistic dose curve algorithm. This kind of correlation seeks the most accurate representation of the data independent of any input from theories arising from the researchers ideas about the underlying fluid mechanics. As such, these correlations provide an objective metric against which observations and other theoretical correlations may be applied. Our correlation is as accurate, or more accurate, than other correlations in the range of Reynolds numbers in which the correlations overlap. However, our formula is not restricted to certain ranges of Reynolds number but instead applies uniformly to all smooth pipe flow data for which data is available. The properties of the classical logistic dose response curve are reviewed and extended to problems described by multiple branches of power laws. This extended method of fitting which leads to rational fractions of power laws is applied to data Marusic and Perry 1995 for the velocity profile in a boundary layer on a flat plate with an adverse pressure gradient, to data of Nikuradse 1932 and McKeon et al. 2004 on friction factors for flow in smooth pipes and to the data of Nikuradse 1933 for effectively smooth pipes.

\section{Introduction}

The goal of this paper is to extract analytic formulas relating the friction factor $\lambda=-d(\mathrm{~d} p / \mathrm{d} x) /\left(\rho \bar{U}^{2} / 2\right)$ to the Reynolds number $R e=\bar{U} d / v$ ( $\bar{U}$ being the average bulk velocity) from processing of data for flow in smooth pipes. No pipe can be perfectly smooth but when the roughness is small enough the flow depends only on the Reynolds number $\lambda=f(R e)$ (and not on roughness) and the pipe is said to be effectively smooth. The effects of roughness on a nominally smooth wall are well described in the following personal communication with Ivan Marusic:

The way I like to consider the effect of roughness vs. a smooth wall is by considering the range of scales in the flow. For a pipe of radius $a$, the friction Reynolds number, $R e_{\tau}=U_{\tau} a / v$, is proportional to the ratio of the largest and smallest length scales in the flow that contribute to the friction factor. The largest length scale is proportional to $a$, and the smallest attached eddies scales with the $v / U_{\tau}$ (say $100 v / U_{\tau}$ and this is towards the wall). (There is also the Kolmogorov scale, but those motions do not contribute to the energy-containing motions in a significant way, especially if the Reynolds number is high enough).

On a smooth wall the range of scales continues to increase unabated as $R e_{\tau}$ increases, and so friction factor is free to change likewise.

On a rough wall the smallest scale is restricted by the roughness scale $k$. That is, as $R e_{\tau}$ increases the smallest scale is fixed by $k$ and will no longer depend on the Reynolds number. For this reason the friction factor levels off and becomes independent of Re. Different roughness scales will cause the friction factor to level off at different values and this leveling off will occur at lower $R e_{\tau}$ for larger values of $k / a$.

An expanded data set for flow in smooth pipes is created by extracting results for effectively smooth pipes from data of Nikuradse 1933 for flows spanning laminar, transition and turbulent flow in rough pipes. New results are obtained by direct comparison of data for smooth pipes with data for effectively smooth 
pipes. We also present results comparing formulas for turbulent flow in smooth pipes based on modifications of classical log laws and the formula anchored in the theory of incomplete similarity (a power law for $\lambda=f(R e)$ with a pre-factor and exponent that also depend on $R e$ ) with each other and with the expanded data set. The principal results of this paper arise from the introduction of a new and systematic method for processing experimental data which can be described fitting data piecewise by linear splines (which are power laws in log-log coordinates). The data in the transition regions between the splines is processed by fitting five points with a logistic dose algorithm. This method of fitting leads to rational fractions of power laws and rational fractions of rational fractions of power laws. It is a fundamentally different than other methods of processing data. Other methods are motivated by flow fundamentals modulated by the researchers' imagination. Our goal is to get formulas of the highest accuracy and greatest range; ideas about fluid mechanics and turbulence do not enter at any stage. We will show that our correlation is as accurate, or more accurate, than other correlations in the range of Reynolds numbers in which the correlations overlap. However, our formula is not restricted to certain ranges of Reynolds number but instead applies uniformly to all smooth pipe flow data for which data is available. Other methods for describing data on turbulent flow in pipes and boundary layers are rooted in hypotheses about the controlling fluid mechanics principles under sway. It can be said that the implementation of the fluid mechanics ideas require a certain number of operational hypotheses leading to functional forms involving unknown quantities which must finally be selected to fit the data. In this sense, all these fluid mechanics motivated fitting methods are semi-empirical. An excellent description of the most popular of these methods has appeared in the review paper of Barenblatt et al. 1997 cited below.

During the more than 60 years of active research into turbulent pipe flow, two contrasting laws for the velocity distribution in the intermediate region have coexisted in the literature (see, eg. Schlichting 1968): the first is the power or scaling law,

$$
\phi=C \eta^{\alpha}
$$

where the $C$ and $\alpha$ are constants (i.e. parameters independent of $\eta$ ) believed to depend weakly on $R e$. Laws such as (1.1) were in particular proposed by engineers in the early years of turbulence research. The second law found in the literature is the universal, Reynolds number independent logarithmic law,

$$
\phi=(\ln \eta) / \kappa+B
$$

where $\kappa$ (von Kármán's constant) and $B$ are assumed to be universal, i.e. Re-independent, constants.

In more recent decades, the logarithmic law (1.2) has been emphasized over the power law (1.1), sometimes even to the exclusion of the latter. The reasons have been mainly theoretical: it was not recognized that the power law has an equally valid theoretical derivation and satisfies the approximate self-consistency (overlap) condition. This theoretical bias has been allowed to obscure the fact that the experimental data unequivocally militate in favor of the power law (1.1)...

It is generally thought that the universal logarithmic law (1.2) is in satisfactory agreement with the experimental data both in pipes and in boundary layers....However, the scaling law (1.1) has also found experimental support, provided the dependence of the quantities $C$ and $\alpha$ on the Reynolds number was properly taken into account. Indeed, Schlichting, following Nikuradse, showed that the experimental data agree with the scaling law over practically the whole cross-section of a pipe...

An important conclusion has been reached: The power law (1.1) and the logarithmic law (1.2) can be derived with equal rigor but from different assumptions. The universal logarithmic law is obtained from the assumption of complete similarity in both parameters $\eta$ and $R e$; physically, this assumption means that neither the molecular viscosity $v$ nor the pipe diameter $d$ influences the flow in the intermediate region. The scaling law (1.1) is obtained from an assumption of incomplete similarity in $\eta$ and no similarity in $R e$; this assumption means that the effects of both $v$ and $\mathrm{d}$ are perceptible in the intermediate region.

\section{Experimental data}

The data we use comes from three sources: (1) the experiments on the flow of water of in smooth pipes of Nikuradse 1932, (2) the experiments of Nikuradse 1933 on water flow in rough pipes from which we have extracted data for "effectively smooth pipes". Figure 1 shows how we have selected effectively smooth pipe 
data from the rough pipe data. Figure 2 shows that the smooth pipe data and effectively smooth pipe data are in good agreement. The third source of data is presented in the paper by McKeon, Swanson, Zagarolla, Donnelly and Smits in 2004 (hereafter MSZDS). Their data for the flow of gases in smooth pipes is presented graphically in figure 3 and in tabular form in table 1 of MSZDS 2004. They note that

The Princeton (Zagarola \& Smits 1998; McKeon et al. 2004a; McKeon, Zagarola \& Smits 2004b) and Oregon (Swanson et al. 2002) research groups have recently examined fully developed pipe flow using very different apparatus. Compressed air is used in the Princeton "Superpipe", whereas the Oregon device uses several room temperature gases: helium, oxygen, nitrogen, carbon dioxide and sulphur hexafluoride are used for relatively small Reynolds numbers, and normal liquid helium (helium I) is used for highest Reynolds numbers. The difference in of the two devices is dramatic: for example, the Superpipe weighs about 25 tons, whereas the Oregon tube weighs about 1 ounce.

Prior to the Princeton and Oregon experiments, the experiments performed by Nikuradse 1932 covered the largest range of Reynolds numbers. Most other experiments span less than an order of magnitude in Reynolds number (see Zagarola 1996, Table 1.2). The data of Nikuradse 1932, 1933 and MSZDS 2004 is relatively easy to process and compare with empirical formulas because it is presented in tabular form.

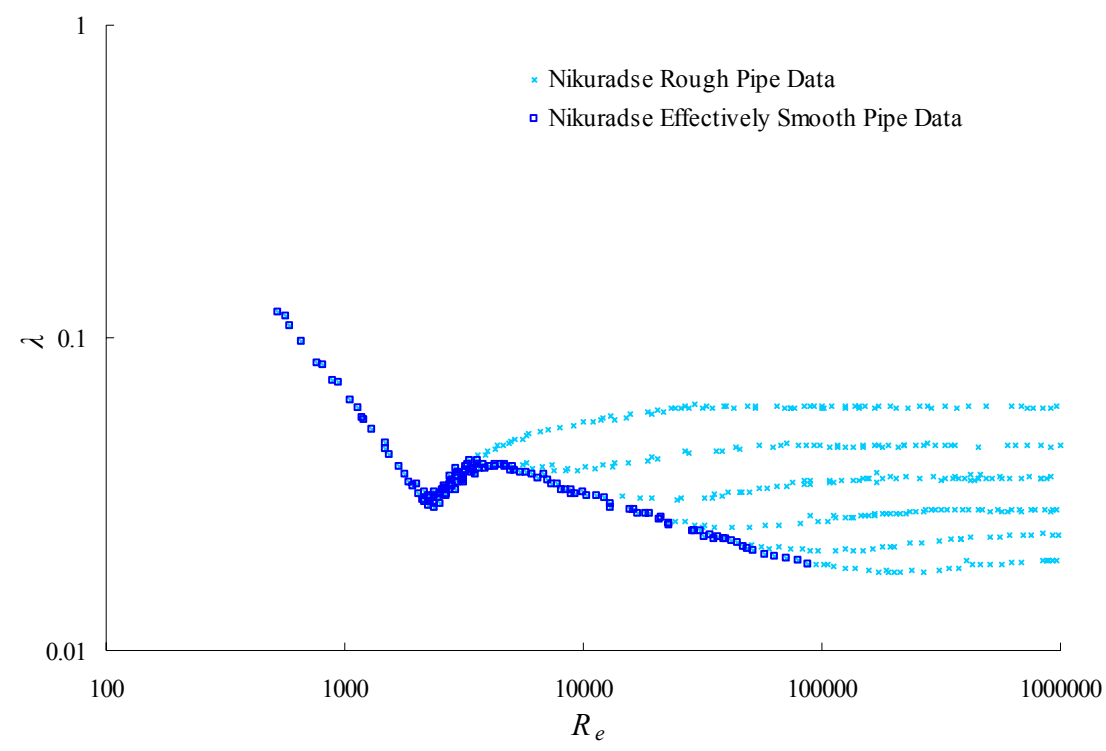

FIGURE 1: Friction factor $(\lambda)$ vs. Reynolds number $(R e)$ in rough pipes (Nikuradse 1933). The dark points on the bottom envelope of curves which depend on roughness do not depend on roughness $\lambda=f(R e)$ can be said to be effectively smooth.

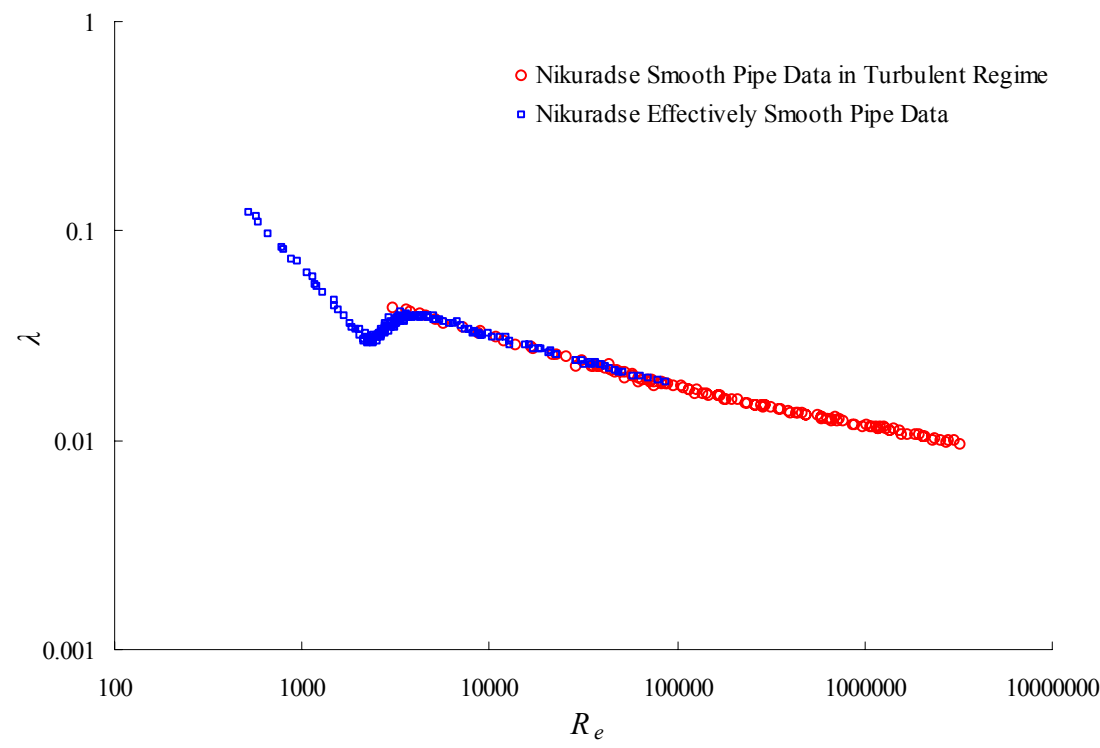

FIGURE $2 . \lambda$ vs. $R e\left(3.1 \times 10^{3}\right.$ $<\operatorname{Re}<3.2 \times 10^{6}$ ) in smooth pipes (open red circles) from Nikuradse 1932 (open blue squares) compared with $\lambda$ vs. $R e$ $\left(5.2 \times 10^{2}<\operatorname{Re}<8.7 \times 10^{4}\right)$ in effectively smooth rough pipes (Nikuradse 1933, see figure 1). Turbulent data for effectively smooth pipes coincides with data from smooth pipes. 


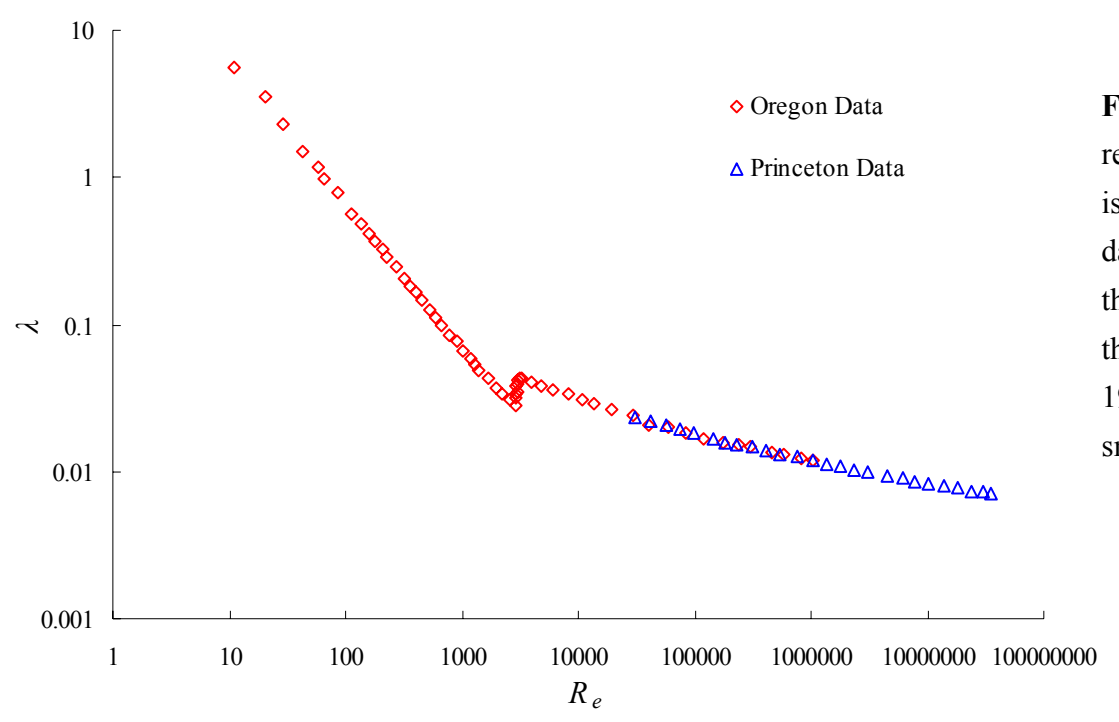

FIGURE 3. $\lambda$ vs. $R e$ from experiments reported in MSZDS 2004. The Princeton data is in good agreement with the smooth pipe data of Nikuradse 1932 (see figure 6.25 in the thesis of McKeon 2003). Figure 4 shows that the Oregon data is not in agreement with the 1933 data of Nikuradse for "effectively smooth" pipes.

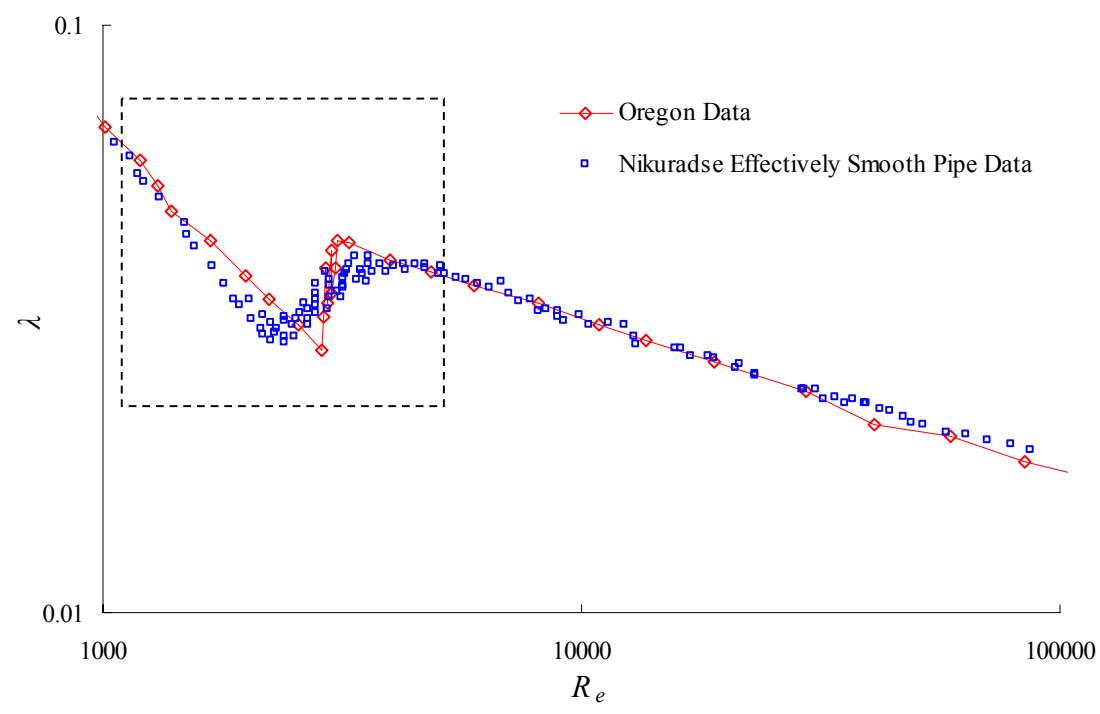

FIGURE 4. Comparison of data from Nikuradse 1933 for effectively smooth pipes with Oregon data (table 1) for flow in smooth pipes. The Oregon data is greatly different than Nikuradse data in the transition region. Data in the transition is associated with the instability of laminar flow which depends on parameters like the pipe length which do not strongly influence laminar or turbulent flow. The data in the transition region forms a cloud rather than a curve.

\section{Comparison of formulas of Barenblatt 2003 and McKeon 2004, 2005 with the data} and each other

The fitting curve proposed by McKeon et al. 2004 is

$$
\frac{1}{\sqrt{\lambda}}=1.930 \log (\operatorname{Re} \sqrt{\lambda})-0.537 ;
$$

it fits Princeton data with a percentage error less than $1.25 \%$ for $31 \times 10^{3}<R e<35 \times 10^{6}$ and $0.5 \%$ for $300 \times 10^{3}<\operatorname{Re}<13.6 \times 10^{6}$.

The curve proposed by McKeon et al. 2005 includes a correction for the viscous deviation from the $\log$ law at the wall and is given by

$$
\frac{1}{\sqrt{\lambda}}=1.920 \log (\operatorname{Re} \sqrt{\lambda})-0.475-\frac{7.04}{(\operatorname{Re} \sqrt{\lambda})^{0.55}} .
$$

This equation predicts the friction factor to within $1.4 \%$ of the Princeton data $(0.6 \%$ at high Reynolds number, $\left.310 \times 10^{3}<\operatorname{Re}<30 \times 10^{6}\right)$ and within $2.0 \%$ of the Blasius relation at low Reynolds numbers $\left(10 \times 10^{3}<\operatorname{Re}<90 \times 10^{3}\right)$.

Barenblatt's 2003 scaling law (8.29) is derived from an extended theory (incomplete similarity) of 
similarity (power law in the Reynolds number) to a form in which the prefactor and exponent of the power law also depend on the Reynolds number; the functional form of the prefactor and exponent are derived from theoretical assumptions. It is given by

$$
\lambda=\frac{8}{\psi^{2 /(1+\alpha)}}
$$

where $\psi=\frac{e^{3 / 2}(\sqrt{3}+5 \alpha)}{2^{\alpha} \alpha(1+\alpha)(2+\alpha)}$ and $\alpha=\frac{3}{2 \ln (\operatorname{Re})}$.

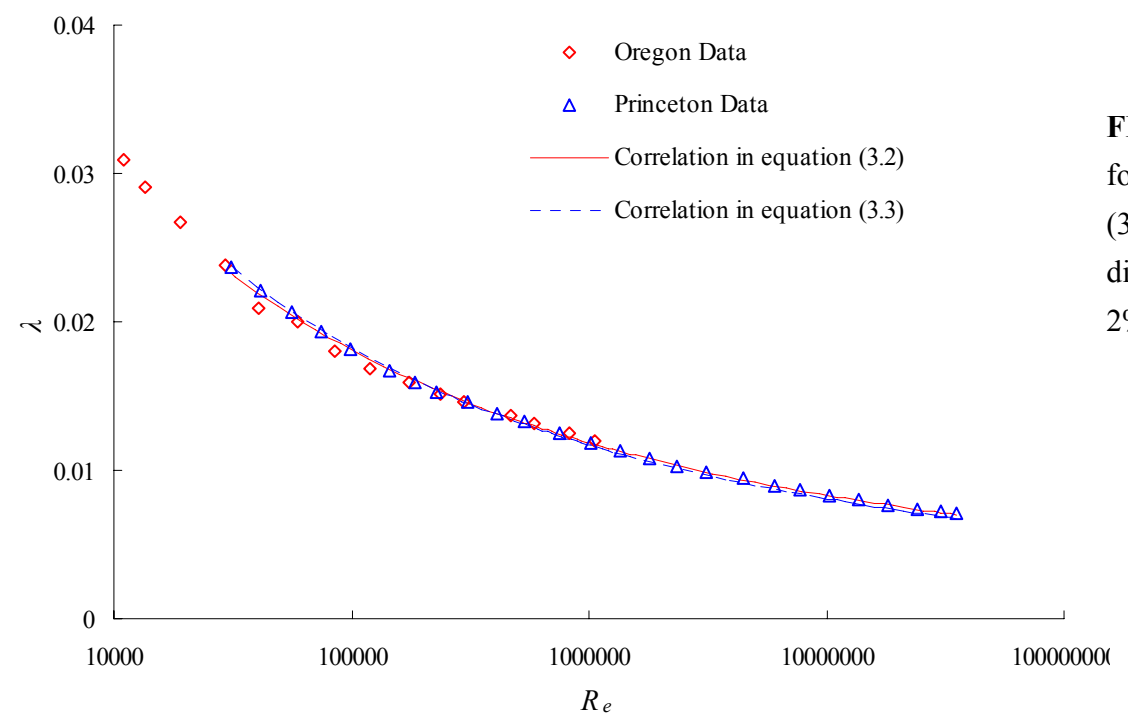

FIGURE 5. Comparison of the power law formula (3.3) and the modified log formula (3.2) with the data of MSZDS. The differences between (3.2) and (3.3) are about $2 \%$ for large $\operatorname{Re}$ (see figure 10 ).

\section{Logistic dose curves for data sets with multiple power law regions}

Prior to the relatively recent work of Joseph and his coworkers, it was not known that the classical logistic dose response curve could be used to fit data arising from real and numerical experiments in fluid mechanics and other hard sciences. In this section we will briefly review the properties of the classical logistic dose response curve and extend this method of fitting for correlations with multiple branches of power laws. This extended method of fitting which leads to rational fractions of power laws and to rational fractions of rational fractions of power laws is applied here to data on the friction factor vs. Reynolds number for laminar, transition and turbulent flow in smooth pipes (McKeon et al. 2004); this method of fitting leads to a composite correlation which describes all the available data unrestricted by Reynolds number. The formula follows from direct processing of the data and does not depend on any assumption or correlation from fluid mechanics.

\subsection{Classical logistic dose response curve}

The logistic function is one of the oldest growth functions and a best candidate for fitting sigmoidal (also known as "logistic") curves. Especially the logistic dose response function is a robust fitting function for transition phenomena. The 3-parameter, 4-parameter and 5-parameter logistic dose response curves are widely used in non-linear data fitting in life sciences; pharmacy and agronomy (see Balakrishnan N. 1992).

A typical 5-parameter logistic dose response curve is given by 


$$
y=a+\frac{(b-a)}{\left[1+\left(\frac{x}{t}\right)^{m}\right]^{n}},
$$

where $x$ and $y$ are the independent and the dependent variables. It is widely used in the literature to describe the correlations between $x$ and $y$ featured by two plateau regions and a transition region. Two typical examples are shown in figure 6.

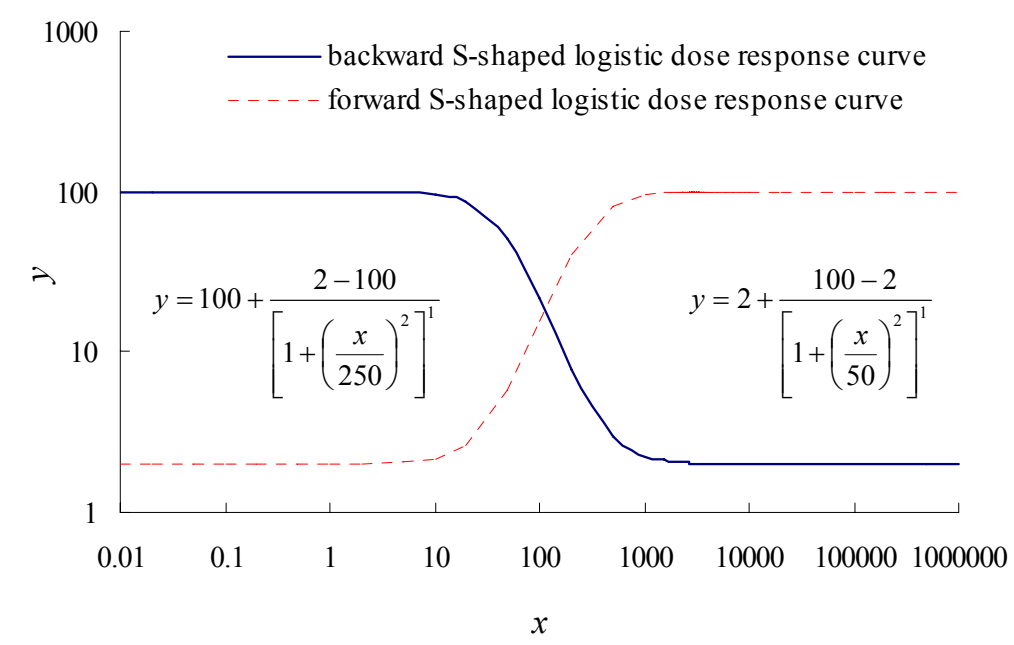

FIGURE 6. Classical forward and backward logistic dose response curves.

To our knowledge the first studies using logistic dose curve fitting in fluid mechanics can be found in the study of sedimentation by Patankar et al. 2002. This study was inspected by R. Barree who explained this method of 5-parameter fitting in an appendix "Fitting power-law data with transition regions by a continuous function: Application to the Richardson-Zaki correlation".

We shall call the correlation of data which can be described by two power laws connected by continuous data in the transition region a two power law correlation (or bi-power correlation). Two power law correlations for sediment transport have been studied by Wang et al. (2003) and for friction factors in turbulent gas-liquid flows by Garcia et al $(2003,2005)$. Correlations of families of bi-power laws depending on a third parameter have been constructed by Viana et al. (2003) who correlated data for the rise velocity of Taylor bubbles in round vertical pipes as a family of bi-power laws of the Froude number vs. Reynolds number indexed by the Eotvos number. A file of papers correlating large data sets from real and numerical experiments can be found at (http://www.aem.umn.edu/people/faculty/joseph/PL-correlations).

The processing of data covered by linear splines and connected by the five point rule of the logistic dose curve (4.1) is an approximate method whose accuracy is judged by analysis of the error of the fit. Moreover, analytical functions like (4.1) which vary on some interval cannot assume a constant value at any finite $\mathrm{x}$ because discontinuous derivatives are required at such points. However, functions with discontinuous derivatives can be closely modeled by analytic functions as in the case in the two logistic dose functions shown in figure 6. The dose function (4.1) is actually a rather complicated nonlinear function and the mathematical problem of approximation of experimental data with such functions deserves further study.

\subsection{Modified logistic dose response curve}

The classical 5-parameter logistic dose response curve (4.1) is modeled to equation (4.2), in which the two constants $a$ and $b$ in equation (4.1) are replaced by two continuous functions $f_{L}(x)$ and $f_{R}(x)$, respectively. $x_{c}, m$ and $n$ are constants. $x_{c}$ is an important constant in determining the convergence trend of $f(x)$ when the coefficient $m$ is negative and $n$ is a small positive number. (Hereafter $x_{c}$ is called the "threshold value" of $x$ ) 


$$
y=f(x)=f_{L}(x)+\frac{f_{R}(x)-f_{L}(x)}{G(x)}=f_{L}(x)+\frac{f_{R}(x)-f_{L}(x)}{\left[1+\left(\frac{x}{x_{c}}\right)^{m}\right]^{n}} .
$$

Here we show how to construct logistic dose curves (see equation (4.2)) for complicated data which can be described by piecewise continuous multiple power laws or multiple rational fractions of power laws, where the rational fractions of power laws themselves are also logistic dose functions. There are three key steps in determining a modified logistic dose curve; these are (1) the selection of two appropriate assembly members $f_{L}(x)$ and $f_{R}(x)$, (2) the estimate of the threshold value $x_{c}$ which identifies the point of intersection of $f_{L}(x)$ and $f_{R}(x)$, and (3) the five-point sharpness control for fitting the transitional part between the two assembly members.

A main idea in the modified logistic dose curve fitting is to force the denominator function $G(x)$ in equation (4.2) to move towards $+\infty$ or 1 rapidly on different sides of the threshold value $x_{c}$ once the independent variable $x$ deviates from $x_{c}$, so that the logistic dose function can approach $f_{L}(x)$ asymptotically on one side and $f_{R}(x)$ on the other side of $x_{c}$. The two assembly members $f_{L}(x)$ and $f_{R}(x)$ can be constants (in this case, it will reduce to the classical logistic dose response curve), power laws, rational fractions of power laws, rational fractions of rational fractions of power laws, or any other type of continuous functions. The splitting trends of $f(x)$ at $x=x_{c}$ is the basis of the sequential construction of a rational fraction of multiple segments of power laws.

\subsubsection{Logistic dose curve fitting for mixed power laws and/or rational fractions of power laws}

The logistic dose function for a two power law correlation (4.2) is a rational fraction of two power laws. Here we go one step further and replace one of the two member functions $f_{L}(x)$ and $f_{R}(x)$ in equation (4.2) with a rational fraction of two power laws. Then we can use the modified logistic dose curve to create a three power law correlation. If both $f_{L}(x)$ and $f_{R}(x)$ are replaced by rational fractions of two power laws, the modified logistic dose curve can even fit data which contains four subsections of power laws. Since $f_{L}(x)$ and $f_{R}(x)$ can be updated with new rational fractions again and again by adding more and more power laws, we may expect to use the modified logistic dose response curve to fit a correlation composed of five or even more branches of power laws. Such correlations lead inevitably to rational fractions of rational fractions of power laws.

\subsubsection{Threshold values $x_{c}$ of the independent variable $x$}

We have found that the independent variable $x$ is sensitive to the threshold value $x_{c}$ in equation (4.2); $x_{c}$ can be used to locate the point of intersection of the two assembly members $f_{L}(x)$ and $f_{R}(x)$. That is to say, $x=x_{c}$ when $f_{L}(x)=f_{R}(x)$. When $x<x_{c}$, the denominator function $G(x)$ in equation (4.2) will approach $+\infty$ and therefore $f(x) \approx f_{L}(x)$ since $m$ is negative. When $x>x_{c}, G(x)$ approaches 1 asymptotically; therefore $f(x) \approx f_{R}(x)$.

\subsubsection{Five-point sharpness control for the transition region between $f_{L}(x)$ and $f_{R}(x)$}

Data which is very closed to the two assembly members $f_{L}(x)$ and $f_{R}(x)$ at the point $x_{c}$ of intersection is said to have sharp transition. The constants $m$ and $n$ can control the smoothness of the transitional part between $f_{L}(x)$ and $f_{R}(x)$. On the transitional part, the smoothness of data increases as the magnitude of $m$ decreases. We use a five-point rule to judge the accuracy of the fitting curve. Five data points on or near the transition segment of the two assembly members are selected; the errors between the five sample points and the fitting curve are calculated and evaluated by the R-square value. The goal of the 
five-point rule is to make the fitting curve for the five selected points as smooth as possible, which can be seen in the zoom-in view of transition region.

\subsubsection{Construction of a logistic dose function for data sets identified by two power laws and a smooth transition segment}

In this section we illustrate the procedure for constructing a logistic dose correlation for two power laws and a smooth transition segment for data of Marusic and Perry (1995) on the variation the dimensionless velocity $\phi=u / u^{*}$ vs the dimensionless distance from the wall $\eta=u^{*} y / v$ in boundary layers with an adverse pressure gradient. An example of the selection of two power laws and the calculation of sharpness control parameters $m$ and $n$ based on raw data is given here. The tabulated results are shown in table 1 for 30 APG mean flow at $R e=19133.02$.

\begin{tabular}{r|c|c|r|r|r|r|r|r|r|r|c}
\hline \multicolumn{10}{c}{ 30 APG MEAN FLOW $(R e=19133.02)$} \\
\hline No. & $\phi$ & $\eta$ & No. & $\phi$ & $\eta$ & No. & $\phi$ & $\eta$ & No. & $\phi$ & $\eta$ \\
\hline 1 & 14.19779 & 39.48126 & 12 & 17.8959 & 204.6324 & 23 & 24.29825 & 916.1413 & 34 & 36.18715 & 2715.102 \\
2 & 14.31916 & 41.87337 & 13 & 18.46791 & 238.6003 & 24 & 25.19049 & 1032.206 & 35 & 37.04935 & 2931.252 \\
3 & 14.48314 & 47.04032 & 14 & 18.71844 & 277.0654 & 25 & 26.06025 & 1157.457 & 36 & 37.60313 & 3147.403 \\
4 & 14.98265 & 54.50369 & 15 & 19.2299 & 320.1233 & 26 & 27.08825 & 1292.467 & 37 & 37.87272 & 3363.554 \\
5 & 15.25863 & 64.45486 & 16 & 19.59897 & 367.9655 & 27 & 28.18673 & 1436.568 & 38 & 37.99084 & 3579.705 \\
6 & 15.62833 & 76.89382 & 17 & 20.22202 & 425.7588 & 28 & 29.35161 & 1590.715 & 39 & 38.02275 & 3795.951 \\
7 & 16.08429 & 91.91626 & 18 & 20.88928 & 484.0305 & 29 & 30.40133 & 1754.718 & 40 & 38.04359 & 3988.087 \\
8 & 16.49045 & 109.4265 & 19 & 21.43574 & 552.0621 & 30 & 31.58338 & 1927.811 & 41 & 38.03168 & 4204.234 \\
9 & 16.84369 & 129.4245 & 20 & 21.99511 & 627.6527 & 31 & 32.73956 & 2110.185 & 42 & 38.04827 & 4363.204 \\
10 & 17.26571 & 151.8146 & 21 & 22.74916 & 714.0556 & 32 & 33.8983 & 2301.65 & 43 & 38.04868 & 4554.573 \\
11 & 17.56293 & 175.7357 & 22 & 23.4404 & 810.1227 & 33 & 35.05426 & 2502.969 & 44 & 38.06908 & 4745.942 \\
\hline
\end{tabular}

TABLE 1. Experimental data (30APG mean flow at $R e=19133.02)$ for boundary layer flows with adverse pressure gradient [Reproduced after Marusic and Perry 1995].

Figure 7 illustrates the typical procedure of the construction of a logistic dose correlation for two power laws connected by a smooth transition. Panel (a) shows all the data from table 1. Five data segments can be identified from the graph: data segments 1, 3, 5 are three power laws segments; segments 2 and 4 are transition segments between power laws. In this example, we do not consider segments 4 and 5. Panel (b) shows two power laws $P_{1}$ and $P_{2}$ which are identified on segments 1 and 3, respectively. The whole plane is divided into four regions by $P_{1}$ and $P_{2}$ (i.e. (1), (2), (3) and (4) in panels (b) and (c)). Panel (b) clearly shows that the transition segment 2 is located in region (1). We shall show that logistic dose function of $P_{1}$ and $P_{2}$ cannot pass through any data points in region (1).

After $P_{1}$ and $P_{2}$ are determined, we can easily obtain the crossing point $\eta=\eta_{c}$ by letting $P_{1}-P_{2}=0$. The two parameters $m$ and $n$ are unknown, and they are obtained by substituting two data points into the following logistic function of $P_{1}$ and $P_{2}$.

$$
\phi=P_{1}(\eta)+\frac{P_{2}(\eta)-P_{1}(\eta)}{\left[1+\left(\eta / \eta_{c}\right)^{m}\right]^{n}} .
$$

- We tentatively choose the point $\mathrm{A}\left(\eta_{1}, \phi_{1}\right)$ which is located right on the power law $P_{1}$ (see panel (b) in figure 7). It follows that 


$$
\phi_{1}=P_{1}\left(\eta_{1}\right)+\frac{P_{2}\left(\eta_{1}\right)-P_{1}\left(\eta_{1}\right)}{\left[1+\left(\eta_{1} / \eta_{c}\right)^{m}\right]^{n}}=\phi_{1}+\frac{P_{2}\left(\eta_{1}\right)-\phi_{1}}{\left[1+\left(\eta_{1} / \eta_{c}\right)^{m}\right]^{n}} .
$$

It follows that $P_{2}\left(\eta_{1}\right)=\phi_{1}$ and $P_{1}=P_{2}$. Only the point of intersection can satisfy this condition but it is not near the transition segment.

- Similarly, if we choose point B $\left(\eta_{2}, \phi_{2}\right)$ which is located on the power law $P_{2}$, we have

$$
\phi_{2}=P_{1}\left(\eta_{2}\right)+\frac{P_{2}\left(\eta_{2}\right)-P_{1}\left(\eta_{2}\right)}{2\left[1+\left(\eta_{2} / \eta_{c}\right)^{m}\right]^{n}}=\phi_{2}+\frac{\phi_{2}-P_{1}\left(\eta_{2}\right)}{\left[1+\left(\eta_{1} / \eta_{c}\right)^{m}\right]^{n}} \text {. }
$$

Therefore $P_{1}\left(\eta_{2}\right)=\phi_{2}$ giving rise to the same problem of point $\mathrm{A}\left(\eta_{1}, \phi_{1}\right)$.

- If we choose a point $\mathrm{C}\left(\eta_{3}, \phi_{3}\right)$ in region (1) but at the left side of $\eta=\eta_{c}$, we obtain

$$
\phi_{3}=P_{1}\left(\eta_{3}\right)+\frac{P_{2}\left(\eta_{3}\right)-P_{1}\left(\eta_{3}\right)}{\left[1+\left(\eta_{3} / \eta_{c}\right)^{m}\right]^{n}}
$$

It follows that

$$
\left[1+\left(\eta_{3} / \eta_{c}\right)^{m}\right]^{n}=\frac{P_{2}\left(\eta_{3}\right)-P_{1}\left(\eta_{3}\right)}{\phi_{3}-P_{1}\left(\eta_{3}\right)}
$$

However, the LHS of equation (4.6) is always positive, but the RHS of (4.6) is negative because $P_{2}\left(\eta_{3}\right)<P_{1}\left(\eta_{3}\right)$ and $\phi_{3}>P_{1}\left(\eta_{3}\right)$. Parameters $m$ and $n$ cannot be found to resolve this contradiction at $\left(\eta_{3}, \phi_{3}\right)$.

- If we choose a point $\mathrm{D}\left(\eta_{4}, \phi_{4}\right)$ in region (1) but at the right side of $\eta=\eta_{c}$, we obtain

$$
\phi_{4}=P_{1}\left(\eta_{4}\right)+\frac{P_{2}\left(\eta_{4}\right)-P_{1}\left(\eta_{4}\right)}{\left[1+\left(\eta_{4} / \eta_{c}\right)^{m}\right]^{n}}
$$

Therefore

$$
\left[1+\left(\eta_{4} / \eta_{c}\right)^{m}\right]^{n}=\frac{P_{2}\left(\eta_{4}\right)-P_{1}\left(\eta_{4}\right)}{\phi_{4}-P_{1}\left(\eta_{4}\right)} .
$$

Since $\phi_{4}>P_{2}\left(\eta_{4}\right)$, we have $0<\left[1+\left(\eta_{4} / \eta_{c}\right)^{m}\right]^{n}<1$. In this case, (4.3) can be satisfied only if the exponent $n$ is negative. However, if $n$ is negative, $\phi$ in equation (4.3) cannot approach the two power laws $P_{1}$ and $P_{2}$ on different sides of $\eta=\eta_{c}$, as expected.

We have shown that the logistic function for power laws $P_{1}$ and $P_{2}$ has no solution if $m$ and $n$ are chosen to match any points in region (1) or on the two power laws.

When transition segment is sharp (i.e. $|m|$ is large and the radius of curvature of the data segment is small), the logistic function (4.3) follows the two power laws closely and the deviation of logistic function from power laws is not even appreciable. In these cases, we do not modify the power laws in data processing because the logistic function can easily satisfy the requirement of fitting error. However, if the radius of curvature of transition segment is large, we want the fitting curve to pass through the data points in a smooth transition. A most convenient way to realize this is to modify the prefactors of the two power laws $P_{1}$ and $P_{2}$. Then we can obtain two new power laws $P_{1}^{\prime}$ and $P_{2}^{\prime}$ (see panel (c)). The purpose of the modification is to move all the data points on transition segment out of region (1). In this case, equation (4.3) can be processed to fit points on the transition segment. This procedure is not sensitive to small changes in the prefactors of $P_{1}^{\prime}$ and $P_{2}{ }^{\prime}$. After $P_{1}^{\prime}$ and $P_{2}{ }^{\prime}$ are determined, we choose two points on or nearby the transition segment and substitute into equation (4.3) to solve for $m$ and $n$ by iterations. In this example, 
$P_{1}, \quad P_{2}, \quad P_{1}^{\prime}$ and $P_{2}^{\prime}$ are $\phi=8.41 \eta^{0.1429}, \phi=1.89 \eta^{0.3726}, \phi=8.76 \eta^{0.1429}$ and $\phi=2.04 \eta^{0.3726}$, respectively. The threshold value $\eta_{c}$ can be determined by letting $P_{1}-P_{2}=0$ and then $\eta_{c}=701.95$. If we choose points No.15 and No.18 (see table 1) to solve for $m$ and $n$, then we can get $m=-0.9225$ and $n=1.0703$. Therefore, we have the composite logistic function expressed as

$$
\phi=P_{1}^{\prime}+\frac{P_{2}^{\prime}-P_{1}^{\prime}}{\left[1+\left(\frac{\eta}{\eta_{c}}\right)^{m}\right]^{n}}=8.76 \eta^{0.1429}+\frac{2.04 \eta^{0.3726}-8.76 \eta^{0.1429}}{\left[1+\left(\frac{\eta}{701.95}\right)^{-0.9225}\right]^{1.0703}}
$$

Equation (4.8) is the rational fraction formula for the data in table 1 without considering the last 10 points.

The correlation in equation (4.8) is shown in panel (d) of figure 7, which passes through the data points in segments 1,2 and 3 perfectly.

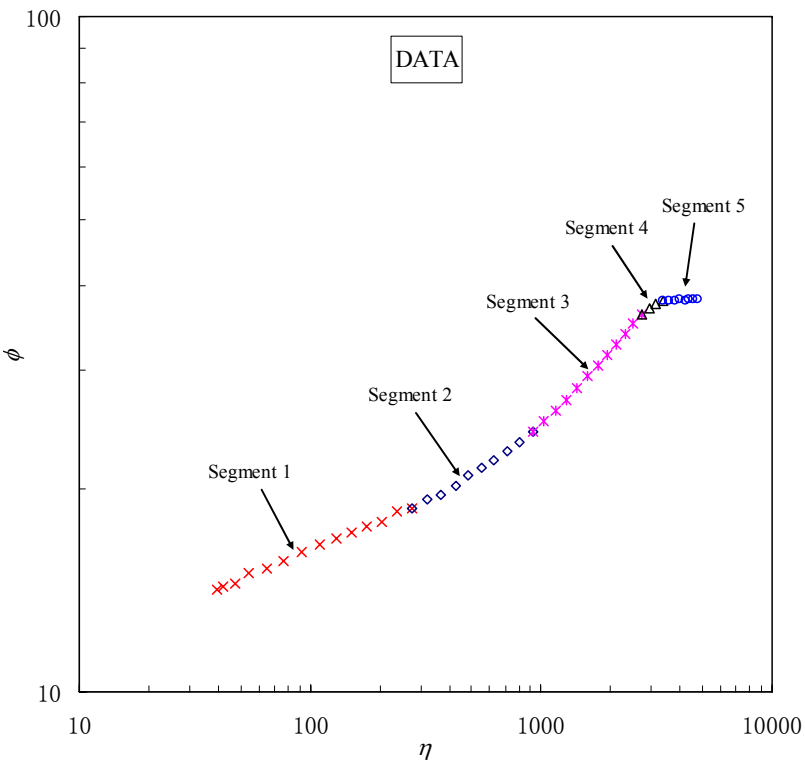

(a)

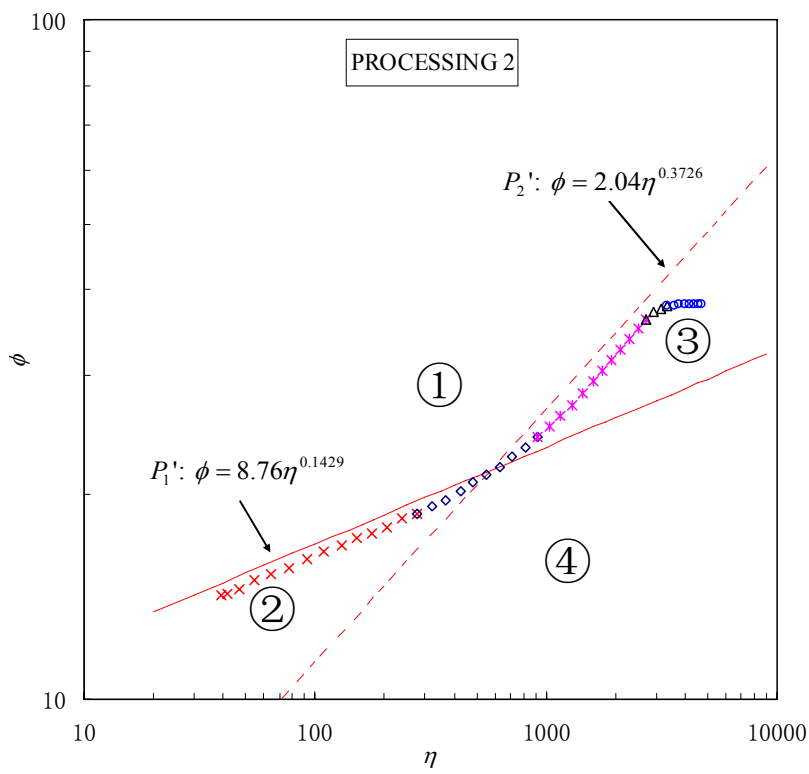

(c)

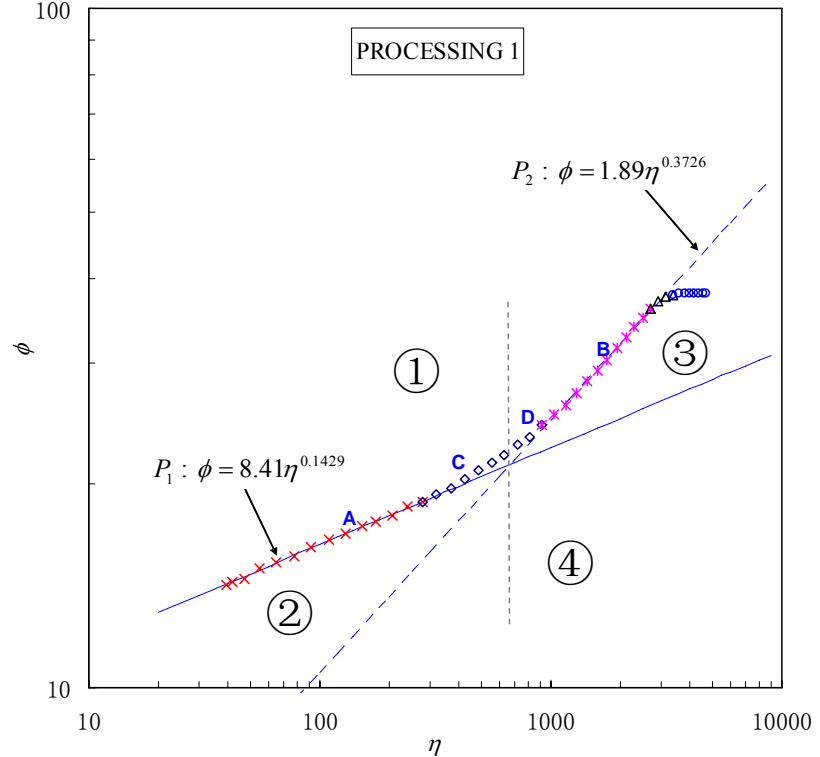

(b)

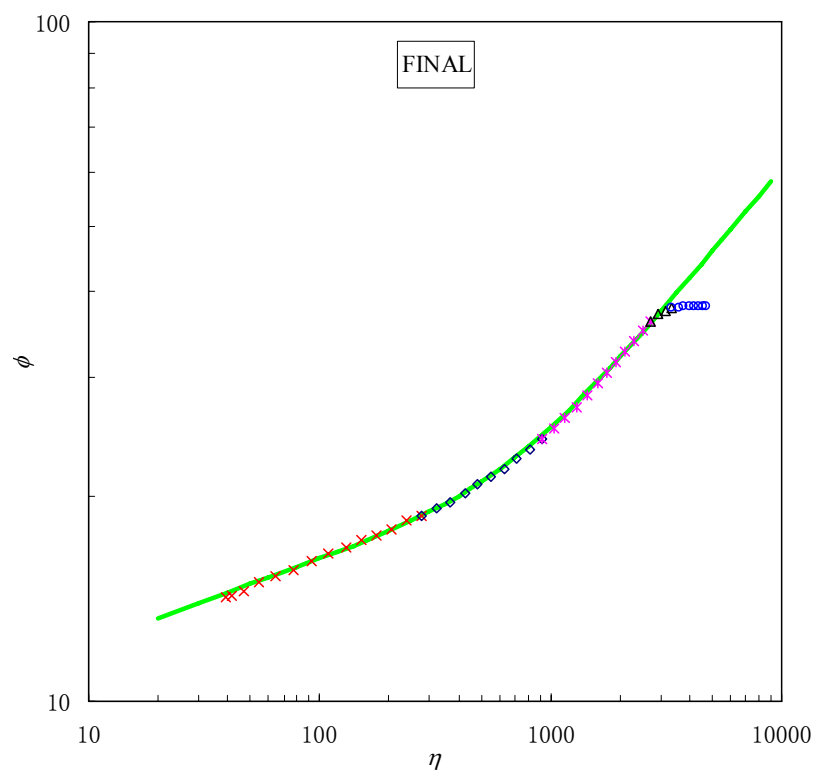

(d)

FIGURE 7. Dimensionless velocity profile vs. dimensionless distance from wall for 30 APG mean flow at $R e=19133.02$. The construction of the logistic dose function as a rational fraction of two power laws is shown in the graphs. The data are from the adverse pressure gradient experiments of Marusic and Perry (1995). The four panels are: (a) the raw data are identified by three 
power law segments and two transition segments, (b) for segments 1 and 3, two power laws $P_{1}$ and $P_{2}$ are identified, (c) $P_{1}$ and $P_{2}$ are modified to $P_{1}^{\prime}$ and $P_{2}^{\prime}$ so that equation (4.3) can be processed to fit the points on or nearby the transition segment, (d) the logistic dose function is constructed as a rational fraction of $P_{1}^{\prime}$ and $P_{2}^{\prime}$ after $m$ and $n$ are determined (the fitting errors for segments 1,2 and 3 are less than $1.9 \%$ or $0.9 \%$ if the first three points are not considered).

Turbulent data suitable for processing as two power law correlations can be found in figures 8.5 and 8.8 in the scaling book of Barenblatt 2003. The correlation in figure 7 corresponds to 1 in figure 8.8 in the Barenblatt book in which the data is described by two broken power laws but no description of the smooth transition between them is given. Keller 2002 introduced a method for constructing a smooth transition between power laws. A differential equation for this problem is proposed and solved. It leads to a power law for the velocity profile followed by a smooth transition to a different power law. Two formulas giving different results are derived; they give different results. Comparisons to data are not given.

\section{Processing the data of MSZDS 2004 for rational fraction correlations between $\lambda$ and $R e$ for laminar, transition and turbulent flow in smooth pipes}

More complicated data which requires the use of more than two power laws may require that the exponents as well as the prefactors need to be modified. The selection of these modifications of the power laws depend on the details of data distribution and cannot be specified a priori. The selection of data points for processing even on apparently smooth transition segments are also uncertain in log-log plots where apparently small errors are actually rather large. If improper data points are chosen, the logistic dose function may not have a solution or may give rise to an inaccurate solution. The construction of accurate logistic dose curve solutions is something of an art. A rule of thumb procedure is to estimate the value of $m$ from the curvature and distribution of data, and then choose only one point from the transition segment to solve for $n$. The processing of multi-power law data is carried out in the appendix, from which we obtain three friction factor correlations $\lambda, \lambda^{\prime}$ and $\lambda^{\prime \prime}$ (see equations (A.5), (A.7) and (A.9) in appendix). $\lambda$ and $\lambda^{\prime}$ are generated by connecting four segments of power laws $\lambda=64 R e^{-1}, \quad \lambda=4.1 \times 10^{-16} R e^{4}, \quad \lambda=0.351 R e^{-0.255}$ and $\lambda=0.118 R^{-0.165}$ from different sequences and are in minute agreement with each other. $\lambda^{\prime \prime}$ is constructed with the addition of the fifth power law $\lambda=19 R e^{-0.82}$ due to the fact that the Oregon data does not agree with the laminar solution $\lambda=64 R e^{-1}$ for $950<R e<2900$. The comparison of $\lambda$ " with the data of MSZDS (2004) is shown in figure 8.

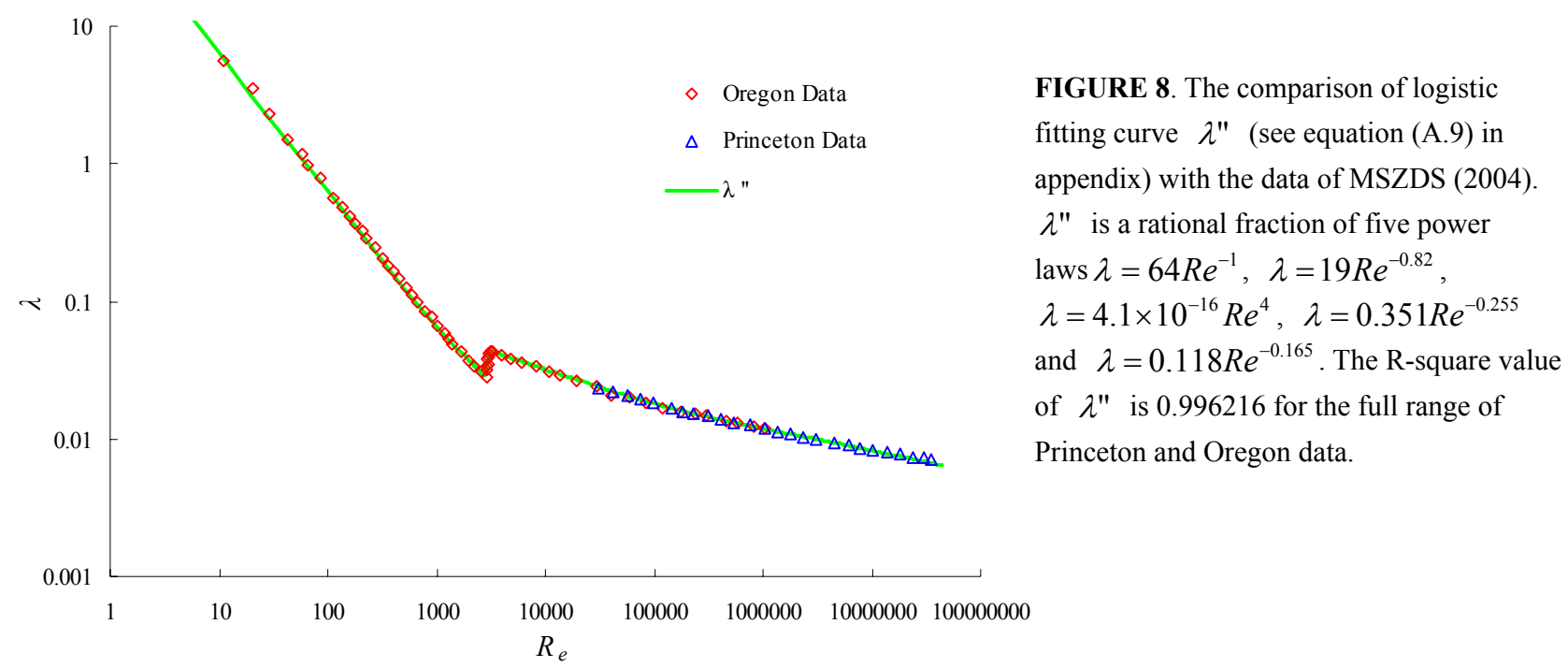




\section{Roughness}

An accurate formula for the full range data of McKeon et al 2004 for pipe flow was derived as a rational fraction of five power laws connected smoothly by the logistic dose curve algorithm. The turbulent flow data is represented by a composition of two power laws with errors less than $1 \%$ for $\operatorname{Re}<13 \times 10^{6}$ but the error increases rapidly thereafter. This rather sudden increase of data can be interpreted as a manifestation of the effect of roughness in an effectively smooth pipe with honed roughness. It is of considerable interest that this critical value was also obtained independently McKeon et al 2004 as a lower bound for the manifestation of roughness by studying the error of their best log formula.

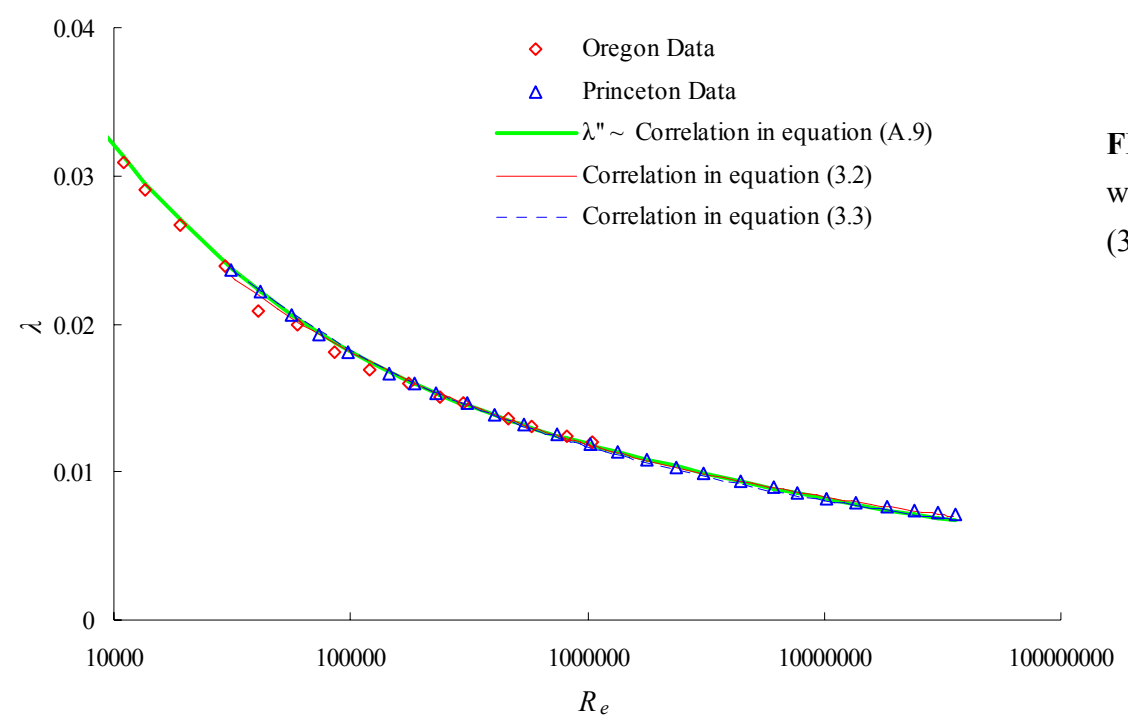

FIGURE 9. Comparison of MSZDS's data with three fitting curves in equations (3.2), (3.3) and equation (A.9) in the appendix.

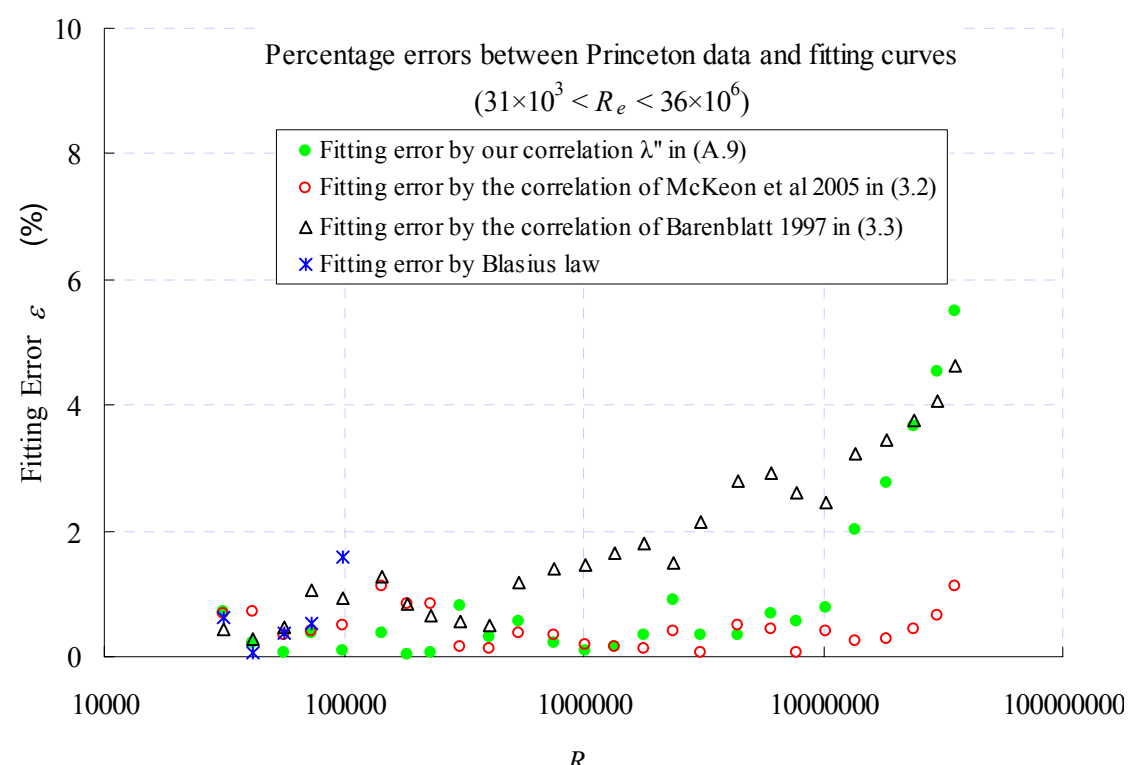

FIGURE 10. Relative fitting error $\varepsilon$ vs. Re between four $\lambda \sim R e$ correlations and the Princeton data in turbulent regime $\left(31 \times 10^{3}<\operatorname{Re}<36 \times 10^{6}\right)$.

We might hope that the quality of our method of logistic dose curve fitting can be improved to arbitrary accuracy, modulo experimental scatter, by choosing more and more branches of power laws and adjusting the sharpness control parameters $m$ and $n$ in equation (A.1). However, as a practical matter, the implementation of fitting more and more power laws is more and more difficult. Moreover, in principle, the five point rule of the logistic dose curve algorithm which is at the center of our fitting method cannot possibly give a perfectly accurate representation of the continuous data connecting power laws. 


\section{Friction factor correlation of Nikuradse's data (1932, 1933) for smooth and effectively smooth pipes}

We conclude our analysis with a comparison of our rational fraction of power law correlation (A.9) with Nikurdses data for smooth and effectively smooth pipes. Equation (A.9) was derived from data of MSZDS 2004 but is in satisfactory agreement with the Nikuradse data. The reader will recall that the full range data given by MSKDS is not in good agreement with the full range data Nikuradse 1933. A new full range correlation which coincides with (A.9) in the overlap region is derived in a companion paper by Yang \& Joseph 2008 on flow in rough pipes is shown figure 12.

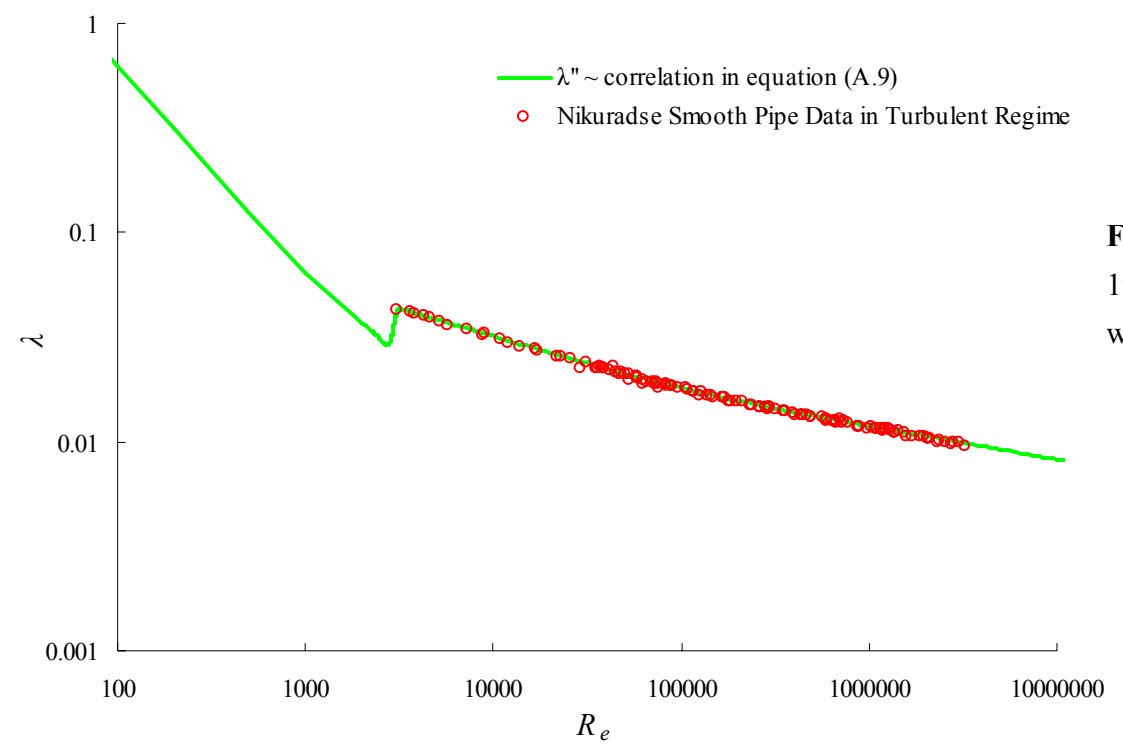

FIGURE 11. Comparison of Nikuradse's 1932 data for turbulent flow in smooth pipes with the correlation $\lambda^{\prime \prime}$ (A.9) in appendix.

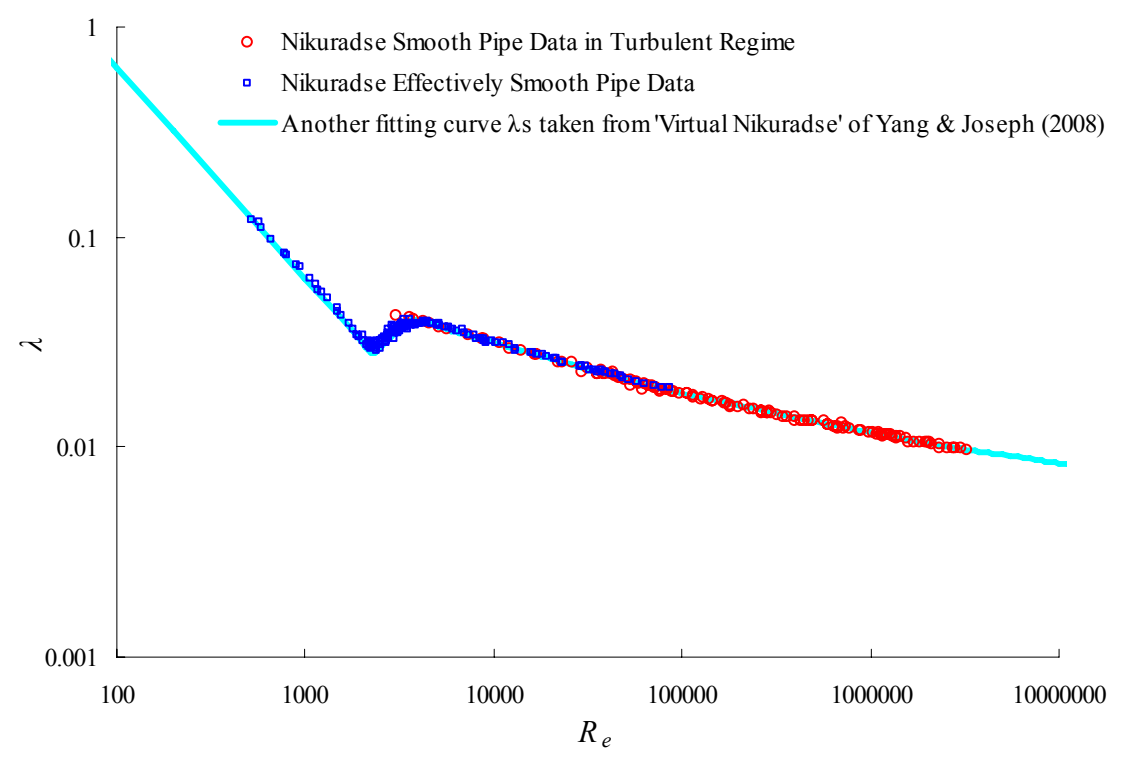

FIGURE 12. Comparison of Nikuradse's 1932 and 1933 data for smooth and effectively smooth pipes with the logistic dose curve $\lambda s$ for effectively smooth pipes derived by Yang \& Joseph 2008. $\lambda s$ is constructed from Nikuradse's 1933 data by a procedure similar to the one described in this paper; $\lambda s$ is a rational fraction of five power laws: $\lambda=64 / \operatorname{Re}$, $\lambda=0.000083 R e^{0.75}, \lambda=0.3164 R e^{-0.25}$, $\lambda=0.1537 R e^{-0.185}$ and $\lambda=0.0753 R e^{-0.136}$. The $\mathrm{R}$-square value of $\lambda s$ is 0.995444 for the full range of Nikuradse's data for smooth and effectively smooth pipes.

\section{Conclusion and discussion}

An effectively smooth pipe is one for which the friction factor depends only on the Reynolds number $\lambda=f(R e)$ and not on roughness. We showed that the bottom envelope of Nikuradse's 1933 data for rough pipes are effectively smooth and in fact coincide with his 1932 data for smooth pipes and with superpipe data presented by McKeon et al. 2004 in the interval $4.2 \times 10^{3}<R e<8.7 \times 10^{4}$. The McKeon data is full range over all Reynolds numbers less than $31 \times 10^{6}$ combining Oregon data for laminar, transition and turbulent flow with Princeton superpipe data for turbulent flow. The Oregon data for transition and near transition 
flows of various gases differ strongly with Nikuradse's 1933 data for the flow of water. We introduced a new method for processing data. The correlation is given as a rational fraction of rational fractions of power laws which is systematically generated by smoothly connecting linear splines in log-log coordinates with a logistic dose curve algorithm. This kind of correlation seeks the most accurate representation of the data independent of any input from theories arising from the researchers ideas about the underlying fluid mechanics. As such, these correlations provide an objective metric against which observations and other theoretical correlations may be applied. Our correlation is as accurate, or more accurate, than best log formula of McKeon et al 2004 and incomplete similarity power law formula of Barenblatt et al 1996 in the range of Reynolds numbers in which the correlations overlap. Moreover, our formula is not restricted to certain ranges of Reynolds number but instead applies uniformly to all smooth pipe flow data for which data is available. Our method was applied to data for the mean flow profile in a boundary layer on a flat plate with adverse pressure gradient. We obtained a correlation formula of good accuracy as a rational fraction of two power laws. A highly accurate formula for the full range data of McKeon et al 2004 for pipe flow was derived as a rational fraction of five power laws connected smoothly by the logistic dose curve algorithm. The turbulent flow data is represented by a composition of two power laws with errors less than $1 \%$ for $R e<13.6 \times 10^{6}$ but the error increases rapidly thereafter. This rather sudden increase of data can be interpreted as a manifestation of the effect of roughness in an effectively smooth pipe with honed roughness. It is of considerable interest, though possibly for tuitis, that this critical value was also obtained independently McKeon et al 2004 as a lower bound for the manifestation of roughness by studying the error of their best log formula.

The ideas and methods presented here may be used to correlate complicated engineering data from real and numerical experiments directly into explicit formulas which could never be obtained by mathematical analysis.

Acknowledgement. This work was supported by the NSF/CTS under grant 0076648 . We have profited from conversations with G. I. Barenblatt, Ivan Marusic, Beverley McKeon and Alex Smits about topics discussed in this paper. 


\section{APPENDIX}

\section{Fitting procedure for constructing multiple power law correlations between $\lambda$ and $R e$ for smooth pipes}

\section{(I). Data for the correlation between $\lambda$ and $R e$ for turbulent flow in smooth pipes}

Now we will fit data for the correlations between the friction factor $\lambda$ and the Reynolds number $R e$. The modified logistic dose response curve can be expressed as

$$
\lambda=f(\operatorname{Re})=f_{L}(\operatorname{Re})+\frac{f_{R}(\operatorname{Re})-f_{L}(\operatorname{Re})}{\left[1+\left(\operatorname{Re} / \operatorname{Re}_{c}\right)^{m}\right]^{n}},
$$

where $R e_{c}$ is the critical Reynolds number (i.e. threshold value of Reynolds number), $f_{L}$ and $f_{R}$ are the two assembly members, $m$ and $n$ are constants.

Experimental data of MSZDS 2004 for friction factors in turbulent flow in smooth pipes is presented in figure 3; the figure shows that the data contains four subsections of straight lines that are distributed in the interval $(0,2900),(2900,3050),(3050,240000)$ and $(240000,+\infty)$, respectively. The four straight lines represent four branches of power laws in the log-log plot shown in figure A1, and they are

$$
f_{a}=64 R e^{-1}, f_{b}=4.1 \times 10^{-16} R e^{4}, f_{c}=0.351 R e^{-0.255} \text { and } f_{d}=0.118 R e^{-0.165} \text {. }
$$

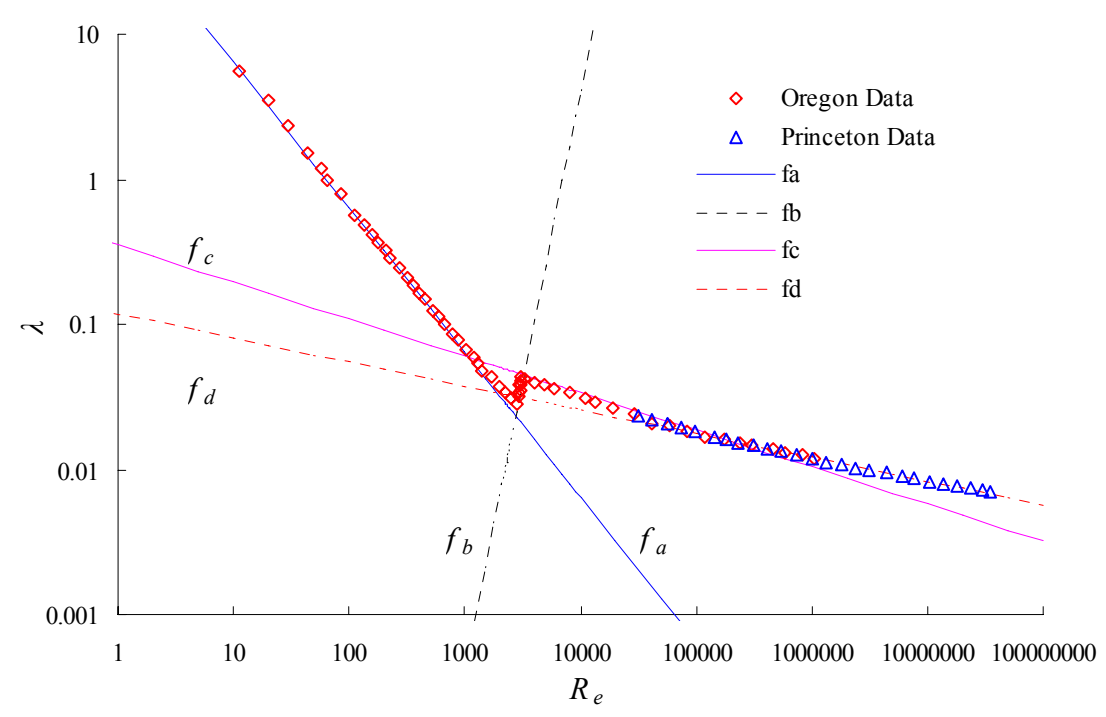

FIGURE A1. Data of MSZDS 2004 for friction factors in laminar, transition and turbulent flow in smooth pipes. Four branches of power laws are identified in the plot, and they are $f_{a}, f_{b}, f_{c}$ and $f_{d}$.

\section{(II). Modified logistic dose curve fitting for two power laws}

Substituting $f_{L}=f_{a}$ and $f_{R}=f_{b}$ into equation (A.1), we obtain a rational fraction for the two subsections $f_{a}$ and $f_{b}$ in figure $\mathrm{A} 1$; identified in figure $\mathrm{A} 2$ by a thick solid line in green color, giving rise to the expression

$$
F_{1}=f_{a}+\frac{\left(f_{b}-f_{a}\right)}{\left[1+\left(\operatorname{Re} / \operatorname{Re}_{c}\right)^{-50}\right]^{0.5}},
$$

where the critical Reynolds number $R e_{c}$ is 2900 . 


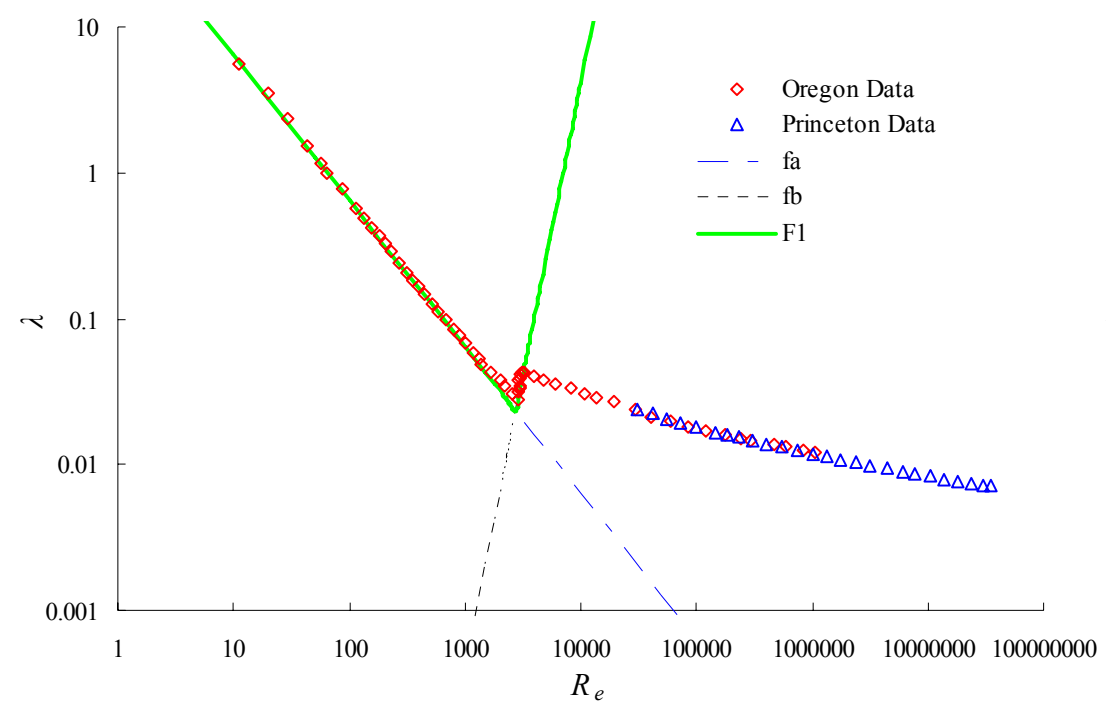

FIGURE A2. The composite logistic dose function $F_{1}$ is a rational fraction of two power laws $f_{a}$ and $f_{b}$ (see equation (A.3))
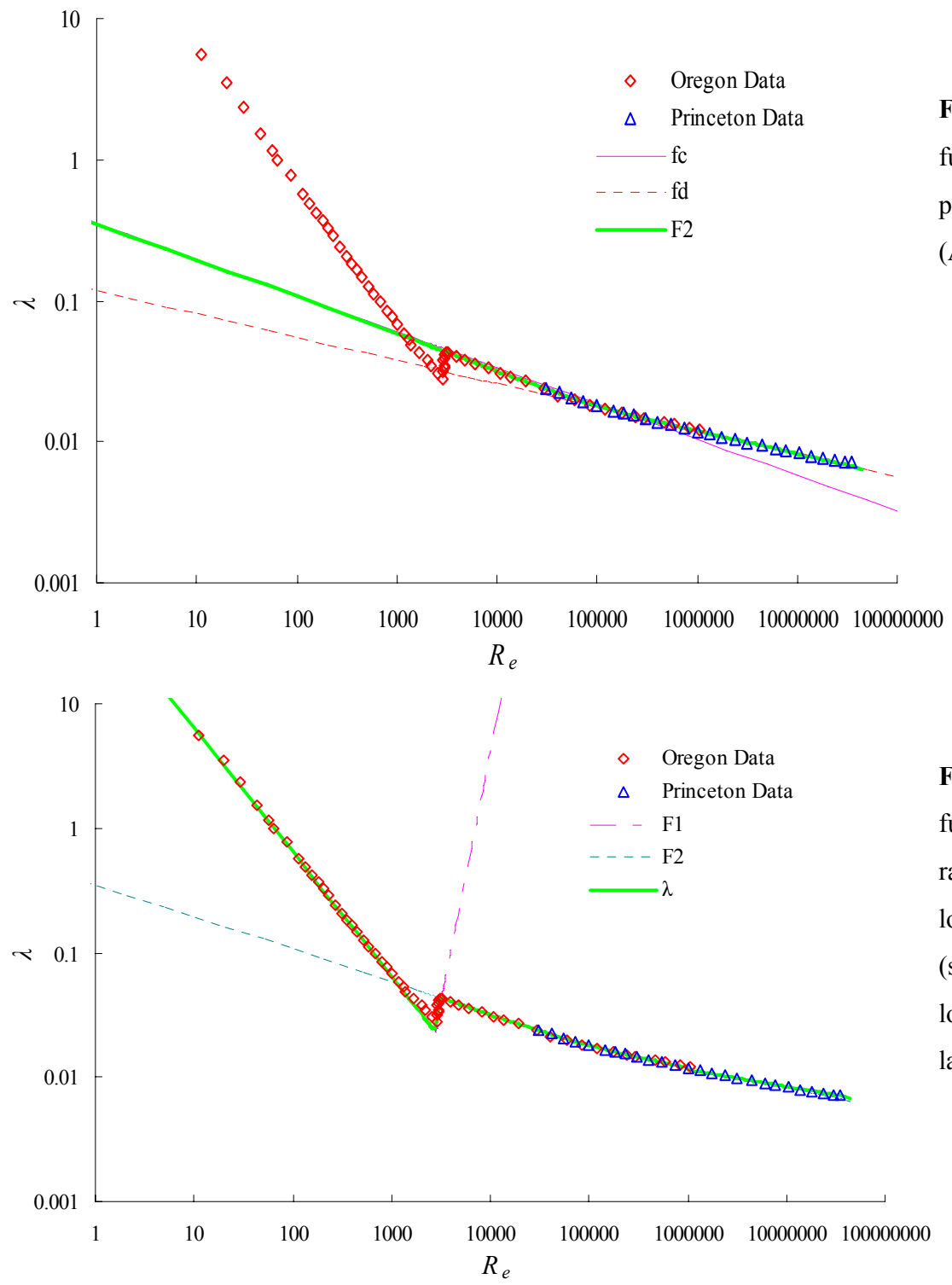

FIGURE A3. The composite logistic dose function $F_{2}$ is a rational fraction of two power laws $f_{c}$ and $f_{d}$ (see equation (A.4))

FIGURE A4. The composite logistic dose function $\lambda$ is a rational fraction of two rational fractions $F_{1}$ and $F_{2}$, which are logistic dose functions of two power laws (see equations (A.3) and (A.4)). $\lambda$ is a logistic fitting curve based on four power laws $f_{a}, f_{b}, f_{c}$ and $f_{d}$.

Similarly, another logistic dose function for the two subsections on the right side is given by

$$
F_{2}=f_{c}+\frac{\left(f_{d}-f_{c}\right)}{\left[1+\left(\operatorname{Re} / \operatorname{Re}_{c}^{\prime}\right)^{-1}\right]^{0.5}},
$$


where the critical Reynolds number $\operatorname{Re}_{c}^{\prime}$ is 240000 (see figure A3).

\section{(III). Logistic dose curve fitting for two rational fractions of power laws}

To describe all of the data of MSZDS 2004 for friction factors in laminar, transition and turbulent flow in smooth pipes connecting the four branches of power laws, we first replace the two assembly members $f_{L}$ and $f_{R}$ with $F_{1}$ and $F_{2}$ which are defined in section (II). This leads to the following rational fraction of power laws:

$$
\lambda=F_{1}+\frac{\left(F_{2}-F_{1}\right)}{\left[1+\left(\operatorname{Re} / \operatorname{Re}_{c}\right)^{-50}\right]^{0.5}},
$$

where the critical Reynolds number $R e_{c}^{\prime \prime}$ is 3050 (see figure A4).

Figure A4 shows that the fitting curve described in equation (A.5) can fit the data of MSZDS 2004 nearly perfectly in the full range of Reynolds numbers.

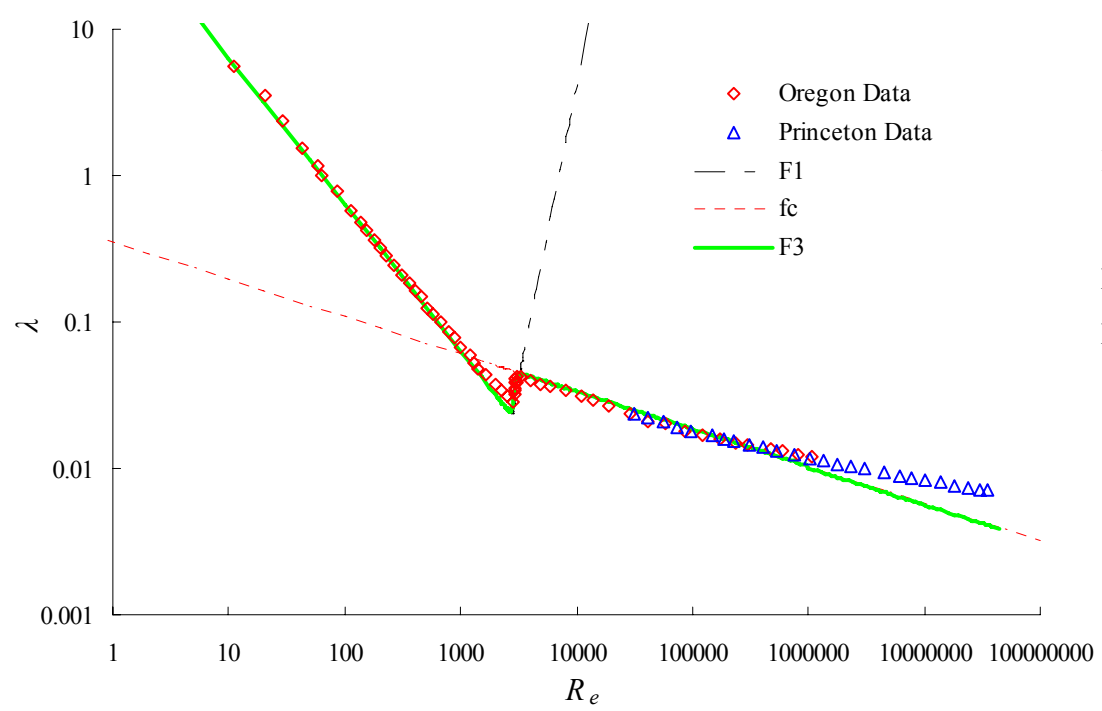

FIGURE A5. The composite logistic dose function $F_{3}$ is a rational fraction of a two power law rational fraction $F_{1}$ and a single power law $f_{c}$ (see equation (A.6)).

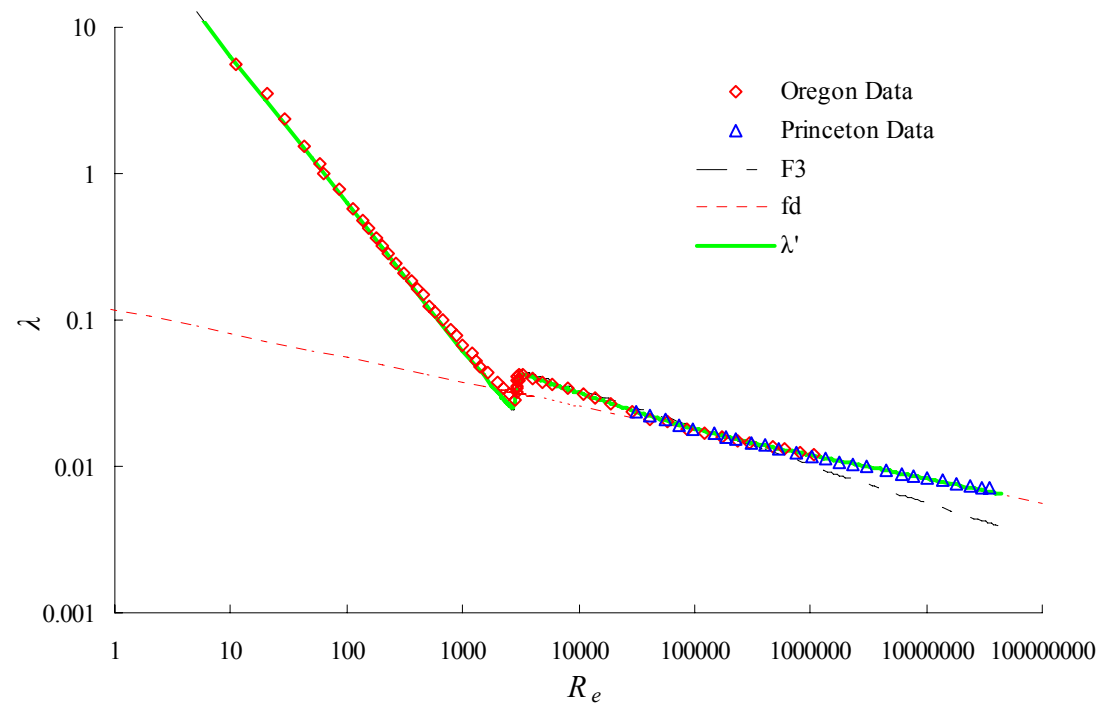

FIGURE A6. The composite logistic dose function $\lambda^{\prime}$ is a rational fraction of a rational fractions $F_{3}$ and a power law $f_{d}$. $F_{3}$ is a logistic dose functions of three power laws (see equation (A.6)). $\lambda^{\prime}$ is a logistic fitting curve based on four power laws $f_{a}, f_{b}, f_{c}$ and $f_{d}$. The R-square value of $\lambda^{\prime}$ is 0.996213 for the full range of Princeton and Oregon data.

Another procedure for fitting two rational fractions of power laws is to construct a logistic dose function, first for a rational fraction of three power laws $f_{a}, f_{b}$ and $f_{c}$ fit to a fourth power law $f_{d}$. This procedure is exhibited in figures A5 and A6. Figure A5 shows the logistic dose curve fitting of three power laws by 
replacing $f_{L}$ and $f_{R}$ in equation (A.1) with $F_{1}$ and $f_{c}$; this curve is expressed as

$$
F_{3}=F_{1}+\frac{\left(f_{c}-F_{1}\right)}{\left[1+\left(\operatorname{Re} / \operatorname{Re}_{c}^{\prime \prime \prime}\right)^{-50}\right]^{0.5}},
$$

where the threshold value of Reynolds number $R e_{c}^{\prime \prime \prime}=3050$.

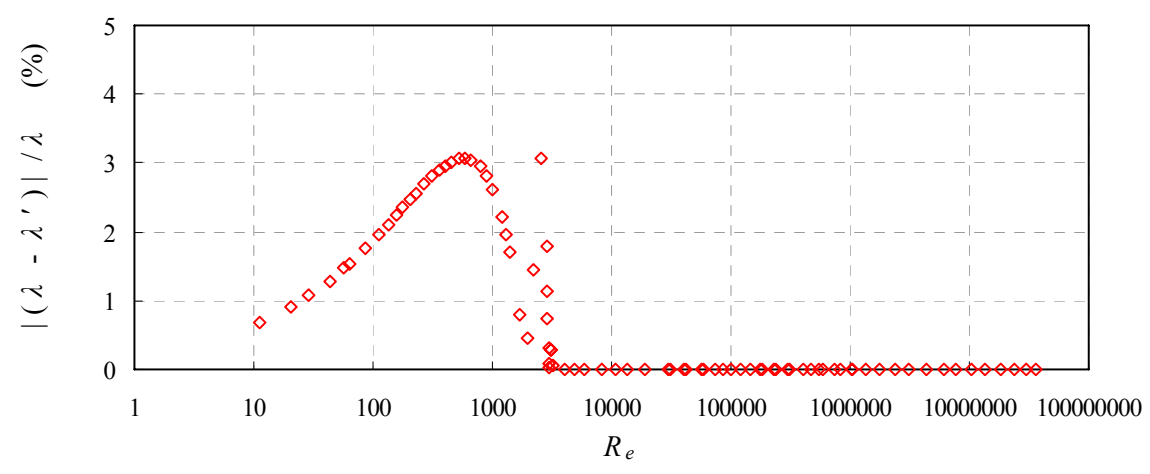

FIGURE A7. Comparison of logistic dose fitting curves $\lambda$ and $\lambda^{\prime}$.

The logistic dose function $F_{3}$ in equation (A.6) represents a fitting curve of three power laws. We can combine $F_{3}$ with the fourth power law $f_{d}$ to obtain the fitting curve for the data of MSZDS 2004 for the full range of Reynolds number (see figure A6). The fitting curve is given by

$$
\lambda^{\prime}=F_{3}+\frac{\left(f_{d}-F_{3}\right)}{\left[1+\left(\operatorname{Re} / \operatorname{Re}_{c} \mathrm{e}^{\prime \prime \prime}\right)^{-1}\right]^{0.5}},
$$

where the threshold value of Reynolds number $R e_{c}^{\prime \prime \prime '}=240000$.

Figures A4 and A6 show that both procedures lead to fitting curves which agree well with the experimental data. Moreover, the two fitting curves in equations (A.5) and (A.7) are in a good agreement with one another. We have compared the difference between the two curves in equations (A.5) and (A.7), and the results are shown in figure A7 which shows that the relative error between the two curves can be controlled within $\pm 3 \%$. The large errors mainly occur in the range of small $R e$ because Oregon data does not agree well with the theoretical solution $\lambda=64 / R e$ for laminar flow, which is used in the constructions of correlations (A.5) and (A.7). Another reason is that the power law $f_{b}$ is too steep in the log-log plot and logistic function may not handle it perfectly.

\section{(IV). Analysis of fitting error $\varepsilon$}

Figures A4 and A6 show that the data does not match the fitting curves perfectly in a small interval around $R e=1000$. The deviation is due to the fact that the data of MSZDS 2004 does not match the laminar correlation $f=64 / R e$. This deviation of the four power law correlations (A.5) and (A.7) from the experimental data can be reduced by representing the data with five rather than four power laws. The measure of the deviation used here is

$$
\varepsilon=\left|\frac{\text { Predicted value by fitting curve - Observed value in experiment }}{\text { Observed value in experiment }}\right| .
$$

The five power law construction is carried out as follows: We first split the laminar correlation $f_{a}=64$ / $R e$ into two parts and replace it with a rational fraction 


$$
\widetilde{f}_{a}=f_{a 1}+\frac{\left(f_{a 2}-f_{a 1}\right)}{\left[1+(\operatorname{Re} / 950)^{-10}\right]^{0.5}}
$$

of two power laws $f_{a 1}=f_{a}=64 / R e$ and $f_{a 2}=19 R e^{-0.82}$. Then the fitting curve (A.7) can be updated to

$$
\lambda "=\widetilde{F}_{3}+\frac{\left(f_{d}-\widetilde{F}_{3}\right)}{\left[1+\left(\operatorname{Re} / \operatorname{Re}_{c}^{\prime \prime \prime}\right)^{-1}\right]^{0.5}},
$$

where $\widetilde{F}_{3}=\widetilde{F}_{1}+\frac{\left(f_{c}-\widetilde{F}_{1}\right)}{\left[1+\left(\operatorname{Re} / \operatorname{Re}_{c}^{\prime \prime \prime}\right)^{-50}\right]^{0.5}}$ and $\widetilde{F}_{1}=\widetilde{f}_{a}+\frac{\left(f_{b}-\widetilde{f}_{a}\right)}{\left[1+\left(\operatorname{Re} / \operatorname{Re}_{c}\right)^{-50}\right]^{0.5}}$.

The fitting curve $\lambda^{\prime \prime}$ in equation (A.9) is shown in figure 8. A zoom-in plot of our fitting curve $\lambda^{\prime \prime}$ for $R e$ ranging from 2000 to 4000 is shown in figure A8; the fitting curve $\lambda^{\prime \prime}$ passes through the data smoothly, the relative errors of some points, due to scatter, cannot be reduced to less than $5-10 \%$.

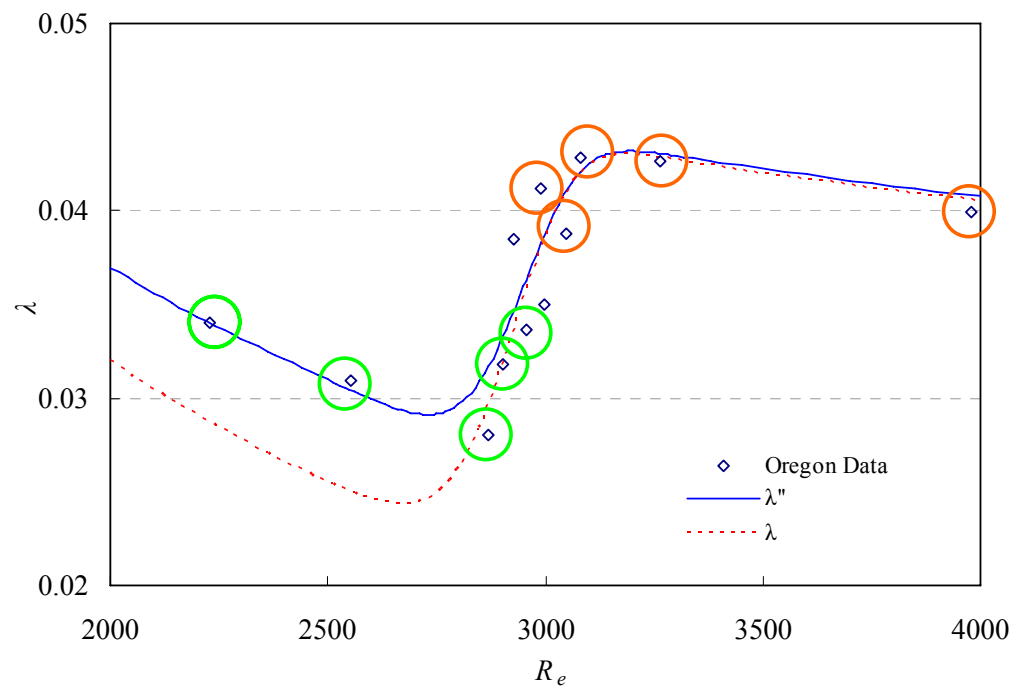

FIGURE A8. The zoom-in view of the modified logistic dose fitting curves $\lambda^{\prime}$ and $\lambda^{\prime \prime}$ for $2000 \leq R e \leq 4000$ shows that the logistic dose fitting curves can be improved by choosing more power laws. The two fitting curves $\lambda^{\prime}$ and $\lambda^{\prime \prime}$ are based on four and five power laws, respectively. The fitting errors on some points cannot be reduced to less than $5-10 \%$ due to the scatter of experimental data. The big circles show the five points which are chosen to fit the data in transition regions.

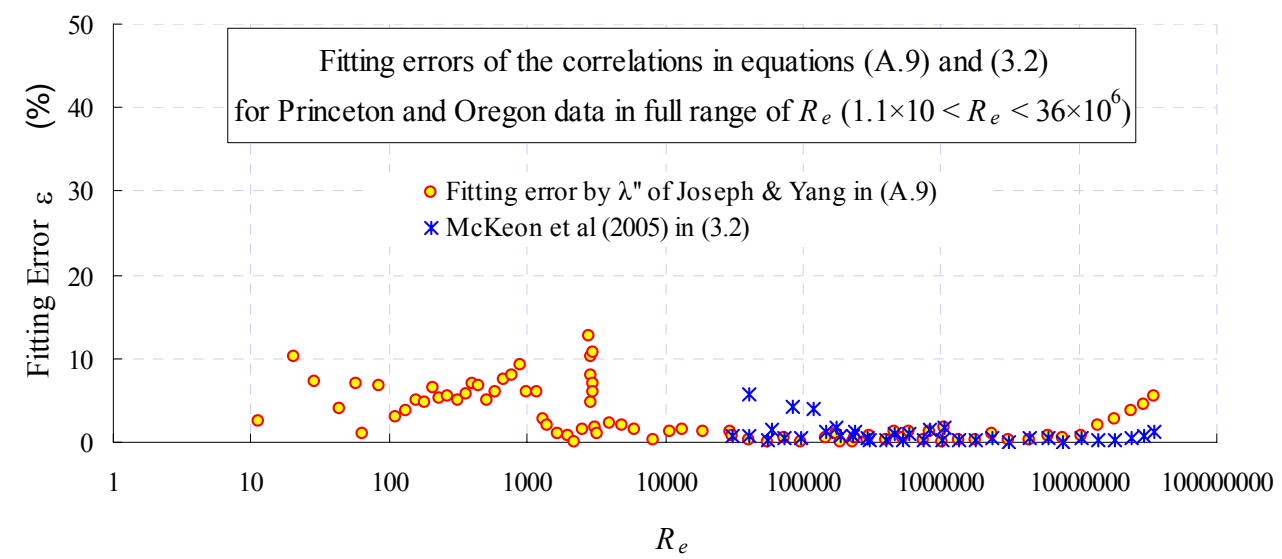

FIGURE A9. Relative fitting error vs. $R e$ between our logistic dose curve $\lambda "$ (A.9), the best formula (3.2) of McKeon et al 2005 and the data of MSZDS 2004. McKeon et al. fit their data with an empirical formula based on logarithms rather than powers; their formula fits the data well in a limited range of Reynolds numbers $\operatorname{Re}\left(31 \times 10^{3} \leq \operatorname{Re} \leq 35 \times 10^{6}\right)$ in the turbulent regime. The relative error of their formula (3.2) in representing the data is also shown in this figure. Our equation (A.9) applies to the full range of Reynolds numbers given by MSZDS 2004 rather than a limited one. 


\section{REFERENCES}

Balakrishnan N. 1992 Handbook of the logistic distribution. New York: Marcel Dekker.

Barendblatt, G. I. 2003 Scaling. Cambridge University Press.

Barenblatt, G. I. \& Chorin, A. J. 1997 Scaling laws for fully developed turbulent flow in pipes. Appl. Mech. Rev., 50(7), 413-429.

Barenblatt, G. I., Chorin, A. J., \& Prostokishin, V. M. 1997 Scaling laws for fully developed flows in pipes: Discussion of experimental data. Proc. Natl. Acad. Sci., 94(3), pp. 773-776.

Garcia, F., Garcia, R., Padrino, J. C., Matta, C., Trallero, J. \& Joseph, D. D. 2003 Power law and composite power law friction factor correlations for laminar and turbulent gas-liquid flow in horizontal pipelines. Int. $J$. Multiphase Flow, 29, 1605-1624.

Garcia, F., Garcia, R. Z. \& Joseph, D. D. 2005 Composite power law holdup correlations in horizontal pipes, Int. J. Multiphase Flow, 31(12), 1276-1303.

MARUŠIC IVAN and PerRY, A. E. 1995 A wall-wake model for the turbulence structure of boundary layers. Part 2. Further experimental support. J. Fluid Mech., 298, 389-407.

McKeon, B. J. 2003 High Reynolds number turbulent pipe flow. Ph.D. thesis, Princeton University

McKeon, B. J., Swanson, C. J., Zaragola, M. V., Donnelly, R. J. \& Smits, A. J. 2004 Friction factors for smooth pipe flow, J. Fluid Mech., 511, 41-44.

McKeon, B. J., Zaragola, M. V. \& Smits, A. J. 2005 A new friction factor relationship for fully developed pipe flow, J. Fluid Mech., 538, 429-443.

NIKURADSE, J. 1932 Laws of turbulent flow in smooth pipes (English translation). NASA TT F-10: 359 (1966).

NIKURADSE, J. 1933 Stromungsgesetz in rauhren rohren, vDI Forschungshefte 361. (English translation: Laws of flow in rough pipes). Technical report, NACA Technical Memorandum 1292. National Advisory Commission for Aeronautics (1950), Washington, DC.

Patankar, N. A., Joseph, D. D., Wang, J., Barree, R., Conway, M. \& Asadi, M. 2002 Power law correlations for sediment transport in pressure driven channel flows, Int. J. Multiphase Flow, 28(8), 1269-1292.

Viana, F., Pardo, R., Yänez, R., TRallero, J. L. \& JosePh, D. D. 2003 Universal correlation for the rise velocity of long gas bubbles in round pipes, J. Fluid Mech., 494, 379-398.

Wang, J., Joseph, D. D., Patankar, N. A., Conway, M. \& Barree, B., 2003 Bi-power law correlations for sedimentation transport in pressure driven channel flows, Int. J. Multiphase Flow, 29(3), 475-494.

YANG, B. H. \& JosePh, D. D. 2008 Virtual Nikuradse, J. Fluid Mech., submitted.

Zagarola, M.V. 1996 Mean flow scaling in turbulent pipe flow. PhD thesis, Princeton University 